\title{
Dynamics and Stability of Bose-Einstein Condensates: the Nonlinear Schrödinger Equation with Periodic Potential
}

\author{
Bernard Deconinck*, Bela A. Frigyik, and J. Nathan Kutz \\ Department of Applied Mathematics, \\ University of Washington, \\ Seattle, WA 98195-2420, USA
}

May 28, 2018

\begin{abstract}
The cubic nonlinear Schrödinger equation with a lattice potential is used to model a periodic dilute gas Bose-Einstein condensate. Both two- and three-dimensional condensates are considered, for atomic species with either repulsive or attractive interactions. A family of exact solutions and corresponding potential is presented in terms of elliptic functions. The dynamical stability of these exact solutions is examined using both analytical and numerical methods. For condensates with repulsive atomic interactions, all stable, trivial-phase solutions are off-set from the zero level. For condensates with attractive atomic interactions, no stable solutions are found, in contrast to the one-dimensional case 8 .
\end{abstract}

\section{Introduction}

The cubic nonlinear Schrödinger equation with repulsive or attractive nonlinearity and an external potential models the mean-field dynamics of a dilute-gas Bose Einstein condensate (BEC) [19, 28]. In this context, the equation is also known as the Gross-Pitaevskii equation. BECs are of interest in the theoretical and experimental physics community since they are examples of macroscopic quantum phenomena which manifest phase coherence [2, 11, 20, 24]. BECs have possible applications in such different areas as quantum logic [7], matter-wave diffraction [25], and matter-wave transport [12].

After rescaling of the physical variables, the governing equation is

$$
i \frac{\partial}{\partial t} \psi(\vec{x}, t)=-\frac{1}{2} \Delta \psi(\vec{x}, t)+\alpha|\psi(\vec{x}, t)|^{2} \psi(\vec{x}, t)+V(\vec{x}) \psi(\vec{x}, t),
$$

where $\Delta$ denotes the Laplacian operator, $\psi(\vec{x}, t)$ is the macroscopic wave function of the condensate in one, two or three dimensions, with $\vec{x}$ defined as $x,(x, y)$ or $(x, y, z)$ respectively. The realvalued function $V(\vec{x})$ is an experimentally generated macroscopic potential. The parameter $\alpha$ determines whether (11) is repulsive ( $\alpha=1$, defocusing nonlinearity), or attractive, $(\alpha=-1$, focusing nonlinearity). The dimensionless variables in (1) are related to the physical variables by $t \rightarrow(4 \pi \hbar|a| N / M \Omega) t, \vec{x} \rightarrow(\Omega / 4 \pi|a| N)^{1 / 2} \vec{x}, \psi \rightarrow(\hbar \Omega)^{-1 / 2} \psi$, and $V(\vec{x}) \rightarrow(M \Omega / 4 \pi|a| N) V(\vec{x})$. Here

\footnotetext{
*Acknowledges support from the National Science Foundation (DMS-0071568)
}

${ }^{\dagger}$ Acknowledges support from the National Science Foundation (DMS-0092682) 


\begin{tabular}{ccccccc}
\hline \hline atom & $a[\mathrm{~nm}]$ & $T_{c}[\mu \mathrm{K}]$ & $N_{c}\left[\times 10^{5}\right]$ & condensate lifetime $[\mathrm{s}]$ & $\Delta t$ & reference \\
\hline \hline${ }^{1} \mathrm{H}$ & 0.072 & 50 & 200,000 & 5 & 3,200 & {$[1]$} \\
${ }^{23} \mathrm{Na}$ & 2.75 & 2 & 7 & 1 & 6,700 & {$[15]$} \\
${ }^{87} \mathrm{Rb}$ & 5.77 & 0.17 & 0.2 & 15 & 2 & {$[3]$} \\
${ }^{87} \mathrm{Rb}$ & 5.77 & 0.43 & 15 & $<25^{*}$ & 74 & {$[2]$} \\
& & & & & & \\
${ }^{7} \mathrm{Li}$ & -1.44 & 0.30 & 1 & $0.1^{\dagger}$ & 0.15 & {$[32]$} \\
${ }^{85} \mathrm{Rb}$ & $-0.9^{\ddagger}$ & 0.006 & 15 & 0.012 & 0.0007 & {$[30]$} \\
\hline \hline
\end{tabular}

Table 1: Physical parameters for various BEC experiments along with the lifetime of the condensate and the elapsed scaled time $\Delta t$ for (1). Note that both ${ }^{7} \mathrm{Li}$ and ${ }^{85} \mathrm{Rb}$ have a negative $s$-wave scattering length which imply an attractive condensate. The last column of the table gives the reference for the corresponding experiment. For the data in the table: $*$ denotes the total time of the experiment, $\dagger$ is estimated from the peak width of Fig. 3 of [32], $\ddagger$ is calculated using the experimental results of 30 .

$N$ is the number of atoms in the volume $\Omega$ containing all atoms (condensed and uncondensed) in the the experiment (typically on the order of $(10 \mu \mathrm{m})^{3}$ [3] $), M$ is the mass of a single atom, and $a$ is the $s$-wave scattering length for collisions between atoms. Depending on the atomic species, $a$ can be either positive or negative so that both signs of $\alpha=\operatorname{sign}(a)$ are relevant for BEC applications. In Table 1, various physical quantities for BEC experiments are displayed. Note that the $s$-wave scattering lengths for ${ }^{7} \mathrm{Li}$ and ${ }^{8} 5 \mathrm{Rb}$ are negative, so these condensates are attractive, whereas the other experiments listed use repulsive condensates.

Current BEC experiments require a confining potential in order to trap the condensate. For theoretical convenience, this confinement is often assumed to be harmonic. Once confined, additional electromagnetic potentials may be imposed on the BEC. In particular, there has been recent interest in confinement of repulsive BECs using standing light waves which results in a sinusoidal potential in the Nonlinear Schrödinger equation (1). Although experiments focus on the quasione-dimensional regime, quasi-two-dimensional and three-dimensional lattice potentials have been suggested [23]. The interaction of the BECs with such potentials is of great practical and theoretical interest. The quasi-one-dimensional regime [10, 27] applies when the longitudinal dimension of the condensate is far greater than the transverse dimensions, which are themselves of the order of the healing length of the condensate. Likewise, the quasi-two-dimensional regime [26] is significant when two dimensions of the condensate are far greater than the third dimension, which is itself of the order of the healing length of the condensate. The healing length is the width of the boundary region over which the probability density of the condensate drops to zero. Thus these quasi-one- or two-dimensional approximations allow for simplification of (11). In reality, however, all condensates are three-dimensional and in many cases no reduction in dimension is justifiable.

In one dimension, the potential generated in experiments is a standing light wave [6] and $V(x)=-V_{0} \sin ^{2}(m x)=\left(\begin{array}{ll}V_{0} \cos (2 m x)-V_{0}\end{array}\right) / 2$. The same potential was considered in [5, 13]. Typically, the condensate is distributed over tens of periods of the standing light wave [1]. In [8, 9, 10], a number of exact solutions of (1) with this potential was constructed and their stability was investigated. Additionally, a more general potential was considered:

$$
V(x)=-V_{0} \operatorname{sn}^{2}(m x, k),
$$

where $\operatorname{sn}(x, k)$ denotes the Jacobian elliptic sine function, with elliptic modulus $k$. It is periodic 


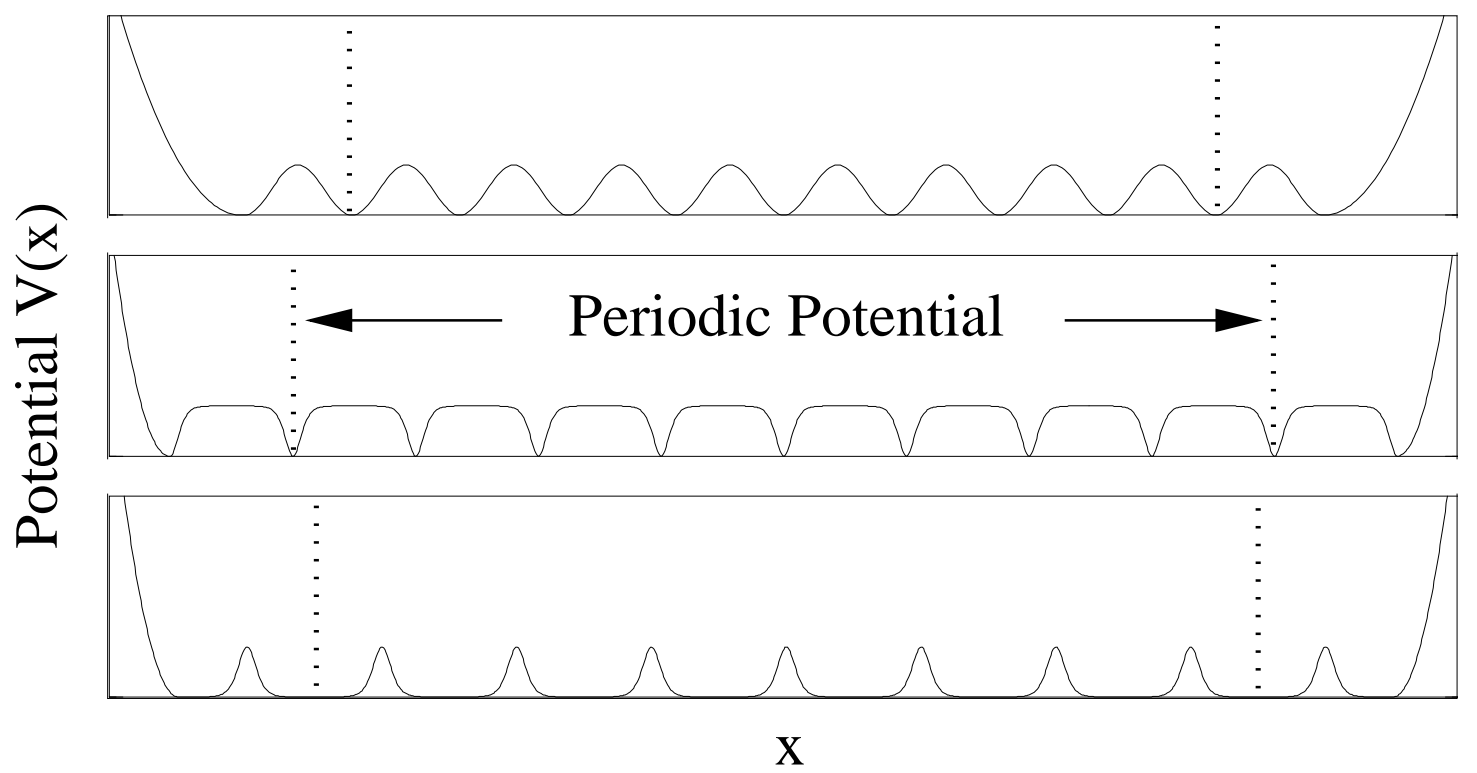

Figure 1: External potential $V(x)$ which exhibits both a periodic and a confining structure. The periodic structure is given by $s n^{2}(x, k)$ which reduces to a standing light wave (as $k \rightarrow 0$ ) in the top figure. Other possibilities of the potential (2) include well-separated troughs or peaks. In this paper, we consider the periodic region away from the edge of the confining experimental potential. Typically this region extends over tens of periods [1].

with period $4 K(k)=4 \int_{0}^{\pi / 2} d \alpha / \sqrt{1-k^{2} \sin ^{2} \alpha}$. This family of potentials contains the standing light wave potential as a special case: $\lim _{k \rightarrow 0} \operatorname{sn}(x, k)=\sin (x)$. This more general potential is considered because it allows the inclusion of different regimes of BEC dynamics. In particular, for values of $k$ up to $k=0.9$, the shape of the potential is virtually indistinguishable from a sinusoidal one, but for values of $k$ close to 1, i.e., $k>0.999$, (2) gives periodic potentials with well-separated peaks or troughs. Figure 1 illustrates the different regimes of the periodic potential (2). The figure also indicates the presence of a confining potential which is applied in all current experiments.

Due to technical complications, there are currently no experiments where a BEC is trapped in a higher-than-one-dimensional periodic potential. However, the interest in the applications mentioned above strongly suggests that these experiments will eventually take place. Already, theoretical investigations of BECs in multidimensional lattice potentials suggest the realization of such experiments 23]. Motivated by the previously referenced developments in BECs, we consider (11) with repulsive and attractive nonlinearity and periodic potential in two and three dimensions. Note that in [16], the repulsive condensate in two-dimensions was investigated.

A judicious choice of the potential allows the construction of a large class of exact solutions, as in one dimension [8, 9, 10, 16]. The family of potentials considered is

$$
V(\vec{x})=-\prod_{i=1}^{d}\left(A_{i} \operatorname{sn}^{2}\left(m_{i} x_{i}, k_{i}\right)+B_{i}\right)+\sum_{i=1}^{d} m_{i}^{2} k_{i}^{2} \operatorname{sn}^{2}\left(m_{i} x_{i}, k_{i}\right),
$$

where $d$ is the number of dimensions: $d=2$ or $d=3$, and $x_{1}=x, x_{2}=y$, and $x_{3}=z$. Here $A_{i}$, $B_{i}$ and $m_{i}, i=1, \ldots, d$ are real constants. The elliptic moduli $k_{i}$ are in the interval $[0,1]$. The 
(a)

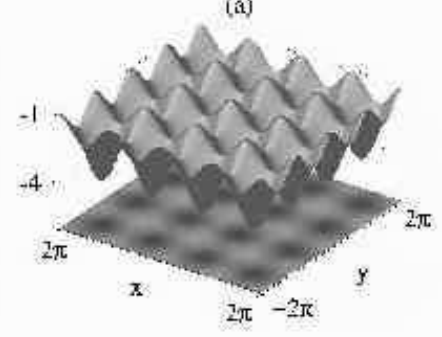

(b)

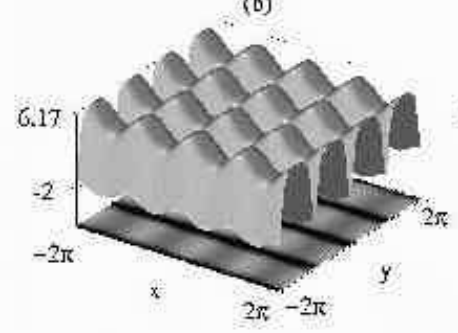

(c)

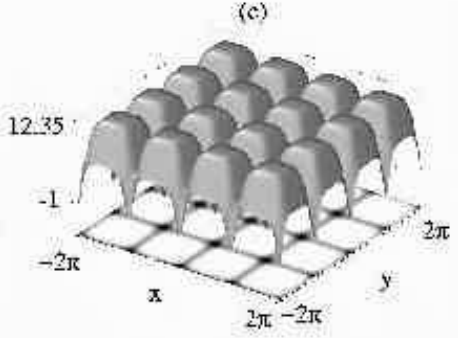

Figure 2: Various lattice potentials. For all figures, $A_{1}=A_{2}=B_{1}=B_{2}=1$. For (a), $k_{1}=k_{2}=0$, $m_{1}=m_{2}=1$; for (b), $k_{1}=0.999, k_{2}=0, m_{1}=2 K(0.999) / \pi, m_{2}=1$. Finally, for (c) $k_{1}=k_{2}=0.999$ and $m_{1}=m_{2}=2 K(0.999) / \pi$.

first term is a straightforward generalization of the one-dimensional potential (2), with an additive constant. The other terms facilitate the construction of exact solutions.

Note that in the important physical case of a trigonometric potential $k_{i} \rightarrow 0, i=1, \ldots, d$, the potential reduces to a product of standing light wave potentials. This generalization of the one-dimensional case is completely analogous with the standard approach of higher-dimensional Kronig-Penney potentials [18]. For non-trigonometric potentials, the exact expression (3) for the potential is necessary to allow the construction of exact solutions. Nevertheless, it is the qualitative features of the potential, i.e., its periodicity and amplitude, that are most important for the stability properties of the solution. As numerical and analytical results throughout this paper demonstrate, the behavior of a solution in a lattice potential is largely independent of the quantitative features of the potential, just as in the one-dimensional case [8, 9, 10]. Figures 2 and 3 display the potential (3) for various values of its parameters in two and three dimensions respectively.

Because of the present lack of experiments in two or three dimensions, this paper can either be read as considering (1) with a periodic potential as a mathematical problem in its own right, or as a collection of predictions from the mathematical mean-field model concerning the dynamics and stability of BECs in higher-dimensional periodic potentials. The paper is arranged as follows: exact solutions of (1]) with periodic potential (3) are constructed. A linear stability analysis is presented for these exact solutions. However, since in many cases the analysis is inconclusive, a complete determination of the stability properties of all our exact solutions is obtained by numerical simulation. We conclude the paper with a brief summary of the results and their implications for BEC dynamics.

\section{Exact solutions}

For typical potentials of (1), the construction of exact solutions is not obvious and usually not possible. For the potentials (2) and (3), it is possible to find families of exact stationary solutions. We look for solutions whose spatial dependence is separated:

$$
\psi(\vec{x}, t)=e^{-i \omega t} \prod_{i=1}^{d} \phi_{i}\left(x_{i}\right) .
$$

Here $d=2$ or $d=3$. The derivation for $d=1$ is similar, but slightly easier. Inserting the ansatz 


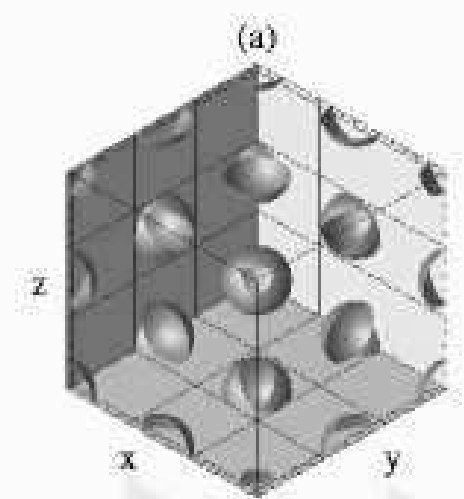

(c)

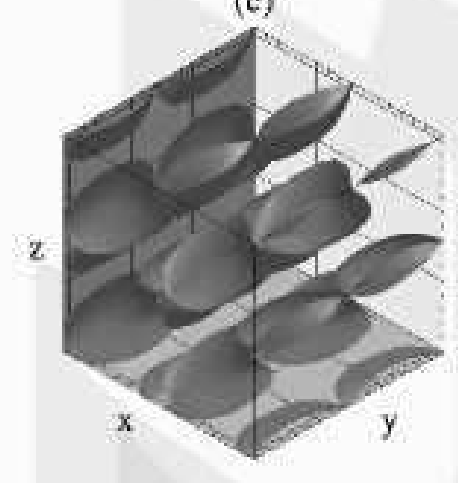

(b)

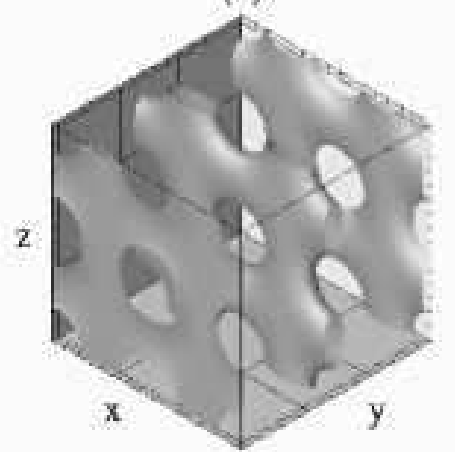

(d)

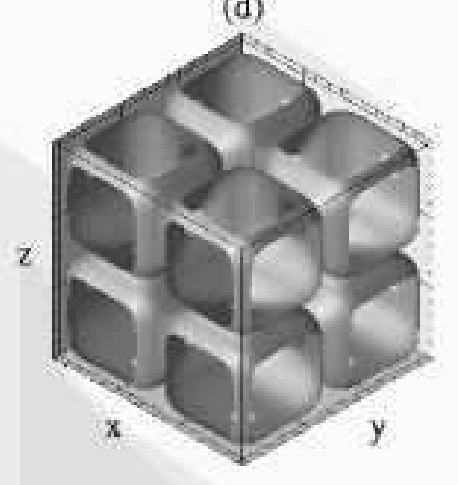

Figure 3: Various 3-D lattice potentials. For all figures, $A_{1}=A_{2}=B_{1}=B_{2}=-1$. For (a), $k_{1}=k_{2}=k_{3}=0$ and $m_{1}=m_{2}=m_{3}=1$; for (b), $k_{1}=k_{2}=0, k_{3}=0.999, m_{1}=m_{2}=1$, $m_{3}=2 K(0.999) / \pi$, for (c), $k_{1}=0, k_{2}=k_{3}=0.999, m_{1}=1, m_{2}=m_{3}=2 K(0.999) / \pi$, for $(\mathrm{d})$, $k_{1}=k_{2}=k_{3}=0.999, m_{1}=m_{2}=m_{3}=2 K(0.999) / \pi$.

(4) into (1) gives

$$
\omega=-\frac{1}{2} \sum_{i=1}^{d} \frac{\phi_{i}^{\prime \prime}}{\phi_{i}}+\alpha \prod_{i=1}^{d}\left|\phi_{i}\right|^{2}+V(\vec{x}),
$$

where prime denotes differentiation with respect to the argument. In order to separate this equation, the potential is written as

$$
V(\vec{x})=-\alpha \prod_{i=1}^{d}\left|\phi_{i}\right|^{2}+\sum_{i=1}^{d} W_{i}\left(x_{i}\right),
$$

which results in the $d$ separated equations

$$
\omega_{i} \phi_{i}=-\frac{1}{2} \phi_{i}^{\prime \prime}+W_{i}\left(x_{i}\right) \phi_{i}
$$

with $\sum_{i=1}^{d} \omega_{i}=\omega$. Using an amplitude-phase decomposition

$$
\phi_{i}\left(x_{i}\right)=r_{i}\left(x_{i}\right) e^{i \theta_{i}\left(x_{i}\right)},
$$


eliminating the overall exponential factor, and equating the imaginary part to zero, gives

$$
\theta_{i}\left(x_{i}\right)=c_{i} \int_{0}^{x_{i}} \frac{d w}{r_{i}^{2}(w)}
$$

where $c_{i}$ is an integration constant. Multiplying the remaining equation by $r_{i}^{\prime}$, results in

$$
\omega_{i} r_{i}^{2}=-\frac{1}{2}\left(r_{i}^{\prime}\right)^{2}-\frac{c_{i}^{2}}{2 r_{i}^{2}}+2 \int W_{i}\left(x_{i}\right) r_{i}\left(x_{i}\right) r_{i}^{\prime}\left(x_{i}\right) d x_{i}
$$

This equation is rewritten using the new function $S_{i}=r_{i}^{2}$. Combining this with a substitution on the integral term finally gives

$$
\left(S_{i}^{\prime}\right)^{2}=-8 \omega_{i} S_{i}^{2}-4 c_{i}^{2}+8 S_{i} \int \tilde{W}_{i}\left(S_{i}\right) d S_{i}
$$

Provided the right-hand side of this equation is a polynomial in $S_{1}$ of degree 3 or 4 , the equation is solvable in terms of elliptic functions [36]. This imposes conditions on the potential for this construction to work: $\tilde{W}_{i}$ as a function of $S_{i}$ is a polynomial of degree at most 2 [36]. Using this result, one finds

$$
r_{i}^{2}=A_{i} \operatorname{sn}^{2}\left(m_{i} x_{i}, k_{i}\right)+B_{i},
$$

which gives rise to the potentials (3). The parameters $\omega, A_{i}, B_{i}, c_{i}$ are constrained by

$$
\begin{aligned}
\omega & =\sum_{i=1}^{d} \omega_{i}=\sum_{i=1}^{d}\left(\frac{1}{2} m_{i}^{2}\left(1+k_{i}^{2}\right)+m_{i}^{2} k_{i}^{2} \frac{B_{i}}{A_{i}}\right), \\
c_{i}^{2} & =m_{i}^{2} \frac{B_{i}}{A_{i}}\left(A_{i}+B_{i}\right)\left(A_{i}+k_{i}^{2} B_{i}\right) .
\end{aligned}
$$

For $d=2$, this results in a one-parameter family of solutions: specifying the potential fixes the parameters $A_{1} A_{2}, B_{1} / A_{1}$, and $B_{2} / A_{2}$. Thus $A_{1} / A_{2}$ is a free parameter. Similarly, for $d=3$, the cross ratio $\left(A_{1}: A_{2}: A_{3}\right)$ is free, resulting in a two-parameter family of solutions. The existence of these solutions requires $r_{i}^{2} \geq 0$ and $c_{i}^{2} \geq 0$. This imposes additional constraints on the parameters: $A_{i} \geq 0, B_{i} \geq 0$, or $B_{i} \geq 0,-A_{i} \leq B_{i} \leq-A_{i} / k_{i}^{2}, i=1, \ldots, d$.

Note that all our solutions satisfy

$$
\psi\left(\vec{x}+2 K\left(k_{i}\right) \overrightarrow{e_{i}}, t\right)=\psi(\vec{x}, t) e^{i \gamma_{i}}, \quad \gamma_{i}=c_{i} \int_{0}^{2 K\left(k_{i}\right)} \frac{d w}{r_{i}^{2}(w)},
$$

where $\overrightarrow{e_{i}}$ is the unit vector in the $x_{i}$ direction. Thus the solutions are nonlinear generalizations of the Bloch states of solid state theory 四]. Note, however, that any such analogy is flawed in principle: the reasoning leading to Bloch functions in solid state theory is based on methods for linear differential equations, whereas the governing equation (11) is nonlinear. Similarly flawed is any interpretation of $\omega$ as an eigenvalue or a separation constant. However, for small amplitude solutions for which the linear terms dominate the nonlinearity, the intuition gained from solid state band-gap theory can be useful in interpreting the weakly nonlinear results.

From (12), it follows that the amplitude $r_{i}\left(x_{i}\right)$ is periodic in $x_{i}$ with period $2 K\left(k_{i}\right) / m_{i}$. However, generally the phase $\theta_{i}\left(x_{i}+2 K\left(k_{i}\right) / m_{i}\right) \neq \theta_{i}\left(x_{i}\right)+2 n \pi$, for some integer $n$. Thus, the solution $\psi(\vec{x}, t)$ is usually not periodic in any of the $x_{i}$, see (15). Imposing periodicity in the $x_{i}$-direction requires 
a quantization of the phase $\theta_{i}\left(x_{i}\right)$. It is unclear if such a quantization in all directions is possible, since not enough free parameters are available to satisfy the number of quantization conditions. There are two special cases in which phase quantization is not a concern. The first case results in trivial-phase solutions, for which $c_{i}=0$. The second case is the trigonometric limit, in which $k_{i}=0, i=1, \ldots, d$.

The solution has trivial phase in the $x_{i}$-direction if $c_{i}$ is zero. There are three possibilities:

$$
\begin{aligned}
B_{i} & =0: & & r_{i}=\sqrt{A_{i}} \operatorname{sn}\left(m_{i} x, k_{i}\right), \\
B_{i} & =-A_{i}: & & r_{i}=\sqrt{-A_{i}} \operatorname{cn}\left(m_{i} x, k_{i}\right), \\
B_{i} & =-A_{i} / k_{i}^{2}: & & r_{i}=\sqrt{-A_{i} / k_{i}^{2}} \operatorname{dn}\left(m_{i} x, k_{i}\right),
\end{aligned}
$$

where $\operatorname{cn}\left(m_{i} x_{i}, k_{i}\right)$ is the Jacobian elliptic cosine function, and $\operatorname{dn}\left(m_{i} x_{i}, k_{i}\right)$ denotes the third Jacobian elliptic function.

The solution is trigonometric in the $i$-direction if $k_{i}$ is zero. Then

$$
r_{i}^{2}(x)=A_{i} \sin ^{2}\left(x_{i}\right)+B_{i}, \quad \tan \left(\theta_{i}\right)=\sqrt{1+\frac{A_{i}}{B_{i}}} \tan \left(m_{i} x_{i}\right),
$$

and phase quantization is satisfied. Notice that it is possible for the solution to have trivial phase in one direction and be trigonometric in the other. In contrast, as $k_{i} \rightarrow 1$ it is possible to obtain solitary wave solutions. These are discussed in [8, 10]. More details on the trigonometric solutions and the trivial phase solutions is also given there.

\section{$3 \quad$ Linear stability}

The linear stability analysis of the solutions found in the previous sections is completely analogous to the one-dimensional case, discussed in [8, 10]. The dimensionality of the solutions only requires minor alterations.

Consider an infinitesimally small perturbation of an exact solution

$$
\psi(\vec{x}, t)=(r(\vec{x})+\epsilon \varphi(\vec{x}, t)) \exp (i \theta(\vec{x})-i \omega t),
$$

with $r(\vec{x})=\prod_{i=1}^{d} r_{i}\left(x_{i}\right)$ and $\theta(\vec{x})=\sum_{i=1}^{d} \theta_{i}\left(x_{i}\right)$ and $r_{i}\left(x_{i}\right), \theta_{i}\left(x_{i}\right)$ defined in (12) and (9) respectively. Substituting this in (11) and linearizing in $\epsilon$ gives

$$
i \frac{\partial \varphi}{\partial t}+\omega \varphi=-\frac{1}{2} \Delta \varphi+\alpha r^{2}\left(\varphi^{*}+2 \varphi\right)+V(\vec{x}) \varphi+\frac{1}{2}(\nabla \theta)^{2} \varphi-\frac{i}{2} \varphi \Delta \theta-i \nabla \theta \cdot \nabla \varphi,
$$

where $\varphi^{*}$ is the complex conjugate of $\varphi, \nabla \theta \cdot \nabla \varphi=\sum_{i=1}^{d} \frac{\partial \theta}{\partial x_{i}} \frac{\partial \varphi}{\partial x_{i}}$, and $(\nabla \theta)^{2}=\nabla \theta \cdot \nabla \theta$. Decomposing $\varphi$ into its real and imaginary parts $\varphi(\vec{x}, t)=u(\vec{x}, t)+i v(\vec{x}, t)$, (19) becomes

$$
\frac{\partial}{\partial t}\left(\begin{array}{l}
u \\
v
\end{array}\right)=J\left(\begin{array}{cc}
L_{+} & -S \\
S & L_{-}
\end{array}\right)\left(\begin{array}{l}
u \\
v
\end{array}\right),
$$

with $J=\left(\begin{array}{rr}0 & 1 \\ -1 & 0\end{array}\right)$ and

$$
\begin{aligned}
L_{+} & =-\frac{1}{2} \Delta+3 \alpha r^{2}+V(\vec{x})-\omega+\frac{1}{2}(\nabla \theta)^{2}, \\
L_{-} & =-\frac{1}{2} \Delta+\alpha r^{2}+V(\vec{x})-\omega+\frac{1}{2}(\nabla \theta)^{2}, \\
S & =-\nabla \theta \cdot \nabla-\frac{1}{2} \Delta \theta .
\end{aligned}
$$


For solutions with non-trivial phase, the analysis of this linear system is currently an open problem. We restrict ourselves to the stability analysis of trivial-phase solutions: $\nabla \theta=0$. Separating the time dependence from the spatial variations,

$$
\left(\begin{array}{l}
u \\
v
\end{array}\right)=\left(\begin{array}{c}
U \\
V
\end{array}\right) e^{\lambda} t
$$

we obtain for trivial-phase solutions

$$
\lambda\left(\begin{array}{c}
U \\
V
\end{array}\right)=J\left(\begin{array}{rr}
L_{+} & 0 \\
0 & L_{-}
\end{array}\right)\left(\begin{array}{l}
U \\
V
\end{array}\right),
$$

with $L_{+}=-\frac{1}{2} \Delta+3 \alpha r^{2}+V(\vec{x})-\omega$ and $L_{-}=-\frac{1}{2} \Delta+\alpha r^{2}+V(\vec{x})-\omega$. Note that the perturbations on the trivial-phase solutions considered do not necessarily have a trivial-phase profile, since $\operatorname{Im}(\varphi)=v$ is not necessarily zero.

Adding the potential $V(\vec{x})$ to the Nonlinear Schrödinger equation destroys three of its four continuous symmetries. However, equation (1) is still phase invariant: the transformation $\psi(\vec{x}, t) \rightarrow$ $e^{i \gamma} \psi(\vec{x}, t)$, with $\gamma$ a real constant, leaves (1) unchanged. Using Noether's theorem,

$$
L_{-} r(\vec{x})=0 .
$$

Thus, $\lambda=0$ is in the spectrum of the Schrödinger operator $L_{-}: 0 \in \sigma\left(L_{-}\right)$. Since both $L_{+}$and $L_{-}$ are self-adjoint operators, their spectrum is contained on the real axis. Furthermore, since $-\frac{1}{2} \Delta$ is a positive operator, and $r(\vec{x}), V(\vec{x})$ are bounded, these spectra are contained in some semi-infinite interval:

$$
\sigma\left(L_{+}\right) \in\left[\lambda_{+}, \infty\right), \quad \sigma\left(L_{-}\right) \in\left[\lambda_{-}, \infty\right),
$$

with $\lambda_{ \pm}=\inf _{\|\eta\|=1}\left\langle\eta\left|L_{ \pm}\right| \eta\right\rangle,\|\eta\|^{2}=\int_{\Omega}|\eta|^{2} d \vec{x}, \Omega=\left\{(x, y) \in\left[0,2 K\left(k_{1}\right)\right] \times\left[0,2 K\left(k_{2}\right)\right]\right\}$ for $d=2$, and $\Omega=\left\{(x, y, z) \in\left[0,2 K\left(k_{1}\right)\right] \times\left[0,2 K\left(k_{2}\right)\right] \times\left[0,2 K\left(k_{3}\right)\right]\right\}$ for $d=3$. Note that since $0 \in \sigma\left(L_{-}\right)$, $\lambda_{-} \leq 0$.

Theorem 1 For condensates with repulsive atomic interactions $(\alpha=1)$ an exact trivial-phase solution such that $r(\vec{x})>0$ is linearly stable.

Proof In this case $\alpha=1$ and $L_{+}=L_{-}+2 r^{2}$. Thus, $\lambda_{+}>\lambda_{-}$. If $r(\vec{x})>0$, then it is the ground state of $L_{-}$[14] and $\lambda_{-}=0, \lambda_{+}>0$. Note that for one-dimensional Schrödinger operators, the ground state is unique. This is not necessarily true for the higher-dimensional case we are considering. Since $L_{+}$is a positive operator, we can construct $L_{+}^{1 / 2}$. Consider

$$
H=L_{+}^{1 / 2} L_{-} L_{+}^{1 / 2}
$$

Let $\xi=L_{+}^{1 / 2} U$, then

$$
\left(H+\lambda^{2}\right) \xi=0 .
$$

The operator $H$ is also self-adjoint. Let $\mu_{0}$ be its smallest eigenvalue, then

$$
\begin{aligned}
\mu_{0} & =\inf _{\|\eta\|=1}\langle\eta|H| \eta\rangle \\
& =\inf _{\|\eta\|=1}\left\langle\eta\left|L_{+}^{1 / 2} L_{-} L_{+}^{1 / 2}\right| \eta\right\rangle \\
& =\inf _{\|\eta\|=1}\left\langle L_{+}^{1 / 2} \eta\left|L_{-}\right| L_{+}^{1 / 2} \eta\right\rangle
\end{aligned}
$$


which implies $\mu_{0} \geq 0$. Note that by letting $\eta=L_{+}^{-1 / 2} r /\left\|L_{+}^{-1 / 2} r\right\|,\left\langle L_{+}^{1 / 2} \eta\left|L_{-}\right| L_{+}^{1 / 2} \eta\right\rangle=0$ so that $\mu_{0}=0$. Thus $\lambda^{2} \leq 0$, and $\lambda$ is imaginary or zero.

Remark As in [10], we have proven the nonexistence of exponentially unstable eigenvalues. However, algebraic instabilities of the zero or other modes are not ruled out.

Theorem 2 For condensates with attractive atomic interactions $(\alpha=-1)$ an exact trivial-phase solution such that $r(\vec{x})>0$ is linearly unstable.

Proof In this case $\alpha=-1$ and $L_{-}=L_{+}+2 r^{2}$. Thus, $\lambda_{-}>\lambda_{+}$. If $r(\vec{x})>0$, then, as above, it is the ground state of $L_{-}\left[14\right.$ and $\lambda_{-}=0, \lambda_{+}<0$. Since $L_{-}$is a positive operator, we can construct $L_{-}^{1 / 2}$. Consider

$$
H=L_{-}^{1 / 2} L_{+} L_{-}^{1 / 2}
$$

Let $\xi=L_{-}^{1 / 2} U$, then

$$
\left(H+\lambda^{2}\right) \xi=0 .
$$

The operator $H$ is self-adjoint. Again, let $\mu_{0}$ be its smallest eigenvalue, then

$$
\begin{aligned}
\mu_{0} & =\inf _{\|\eta\|=1}\langle\eta|H| \eta\rangle \\
& =\inf _{\|\eta\|=1}\left\langle\eta\left|L_{-}^{1 / 2} L_{+} L_{-}^{1 / 2}\right| \eta\right\rangle \\
& =\inf _{\|\eta\|=1}\left\langle L_{-}^{1 / 2} \eta\left|L_{+}\right| L_{-}^{1 / 2} \eta\right\rangle \\
& <0 .
\end{aligned}
$$

Thus there is at least one $\lambda^{2}$ such that $\lambda^{2}>0$, resulting in a pair of opposite, real $\lambda^{\prime}$ 's.

For some other cases, more can be said about the stability or instability of an exact solution, as in [10]. However, those stability criteria are not as straightforward to apply as the two given here, and we only make use of the two criteria above.

It follows from our derivation of (24) that the solution which has a $\operatorname{dn}\left(m_{i} x_{i}, k_{i}\right)$ function in all directions is a deformation of the ground state of the linear Schrödinger equation with periodic potential given by (3). Since (1) is nonlinear, the concept of a ground state as an eigenfunction corresponding to the lowest eigenvalue as used in quantum mechanics has to be abandoned. However, the ground state can still be defined as the global minimizer (if one exists) of the Hamiltonian of the equation, with the constraint $\|\psi\|^{2}=C$, for some constant $C$. This Hamiltonian is

$$
\left.\mathcal{H}(\psi)=\int_{\Omega}\left(\frac{1}{2} \nabla \psi \cdot \nabla \psi^{*}+V(\vec{x})|\psi|^{2}+\frac{1}{2} \alpha|\psi|^{4}\right)\right) d \vec{x} .
$$

It is bounded from below only for condensates with repulsive interactions. Appendix A (due to Jared C. Bronski) demonstrates that in this case, the $\operatorname{dn}\left(m_{i} x_{i}, k_{i}\right)$ solution is indeed the ground state of the nonlinear equation. Since the Hamiltonian (30) is not bounded from below for condensates with attractive interactions, no such ground state exists for this case. 


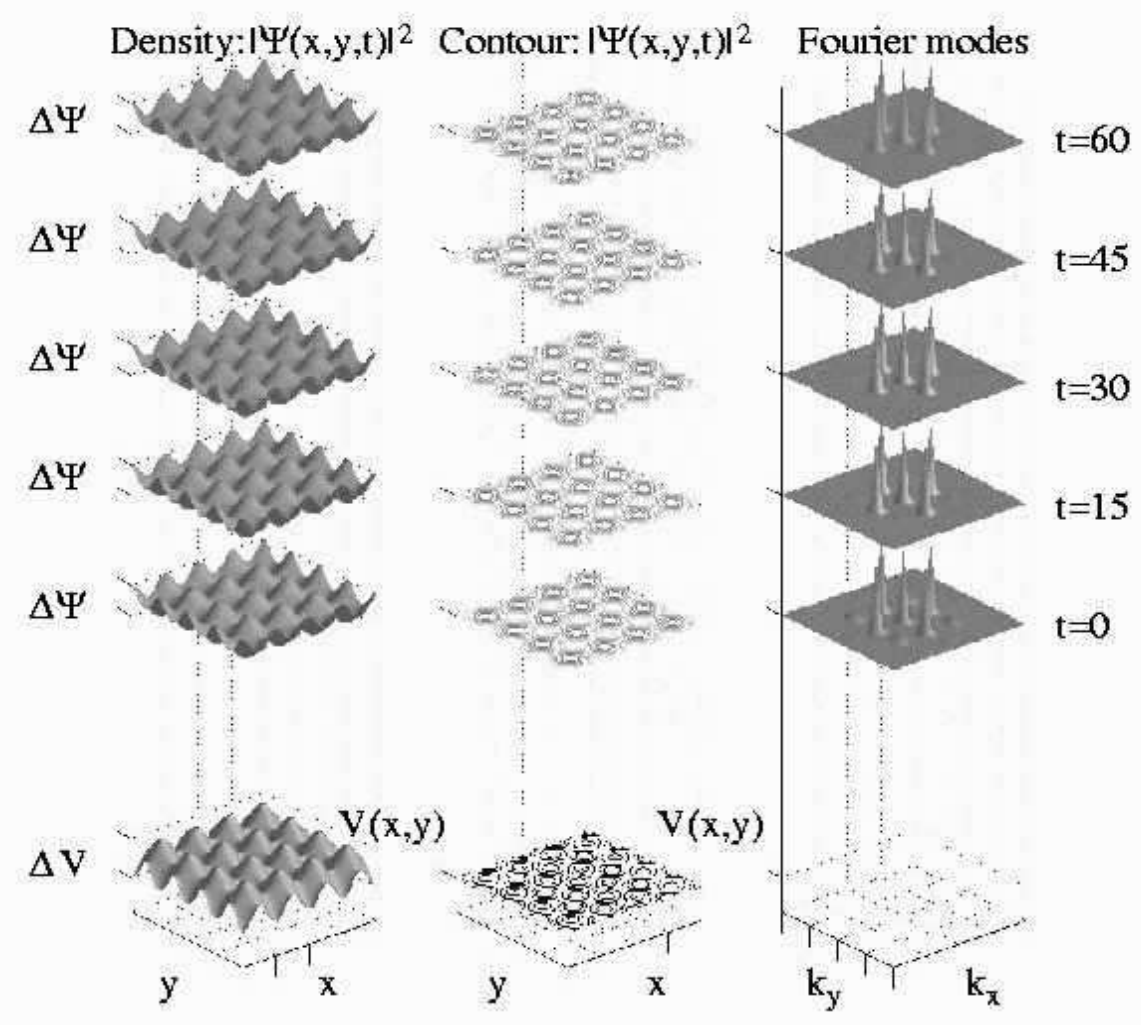

Figure 4: The two-dimensional stable repulsive evolution of the $\operatorname{dn}\left(m_{i} x_{i}, k_{i}\right)$ solution corresponding to (160) over four periods, with $k_{1}=k_{2}=0.5, m_{1}=m_{2}=1$, and $A_{1}=A_{2}=-1$.

\section{Condensates with repulsive interaction}

In this section, the numerical solutions of (1) in the repulsive regime of the nonlinear Schrödinger equation are considered. The initial conditions are selected from the exact solutions given by (4) and (12-14). Of particular interest are the trivial-phase solutions given by (16a-c) and the nontrivial phase solutions in the trigonometric limit (17). These solutions automatically satisfy the phase quantization condition for our periodic domain [8, 10] and correspond to a solution with a ramped phase profile. The numerical procedure used is a fourth-order Runge-Kutta method in time and a filtered pseudospectral method in space [22]. For each experiment, a small amount of white noise was added to the initial conditions as a perturbation to expedite the onset of any instability.

\subsection{Numerical Simulations: Square, Regular Lattice}

Our computational studies begin by considering the case of a square, regular lattice. Thus the spatial domains of all $x_{i}$ are identical as are the elliptic moduli $k_{i}$, and the generated lattice potential $V(\vec{x})$ and the solution $\psi(\vec{x}, t)$ do not distinguish between the different directions $x_{i}$. 


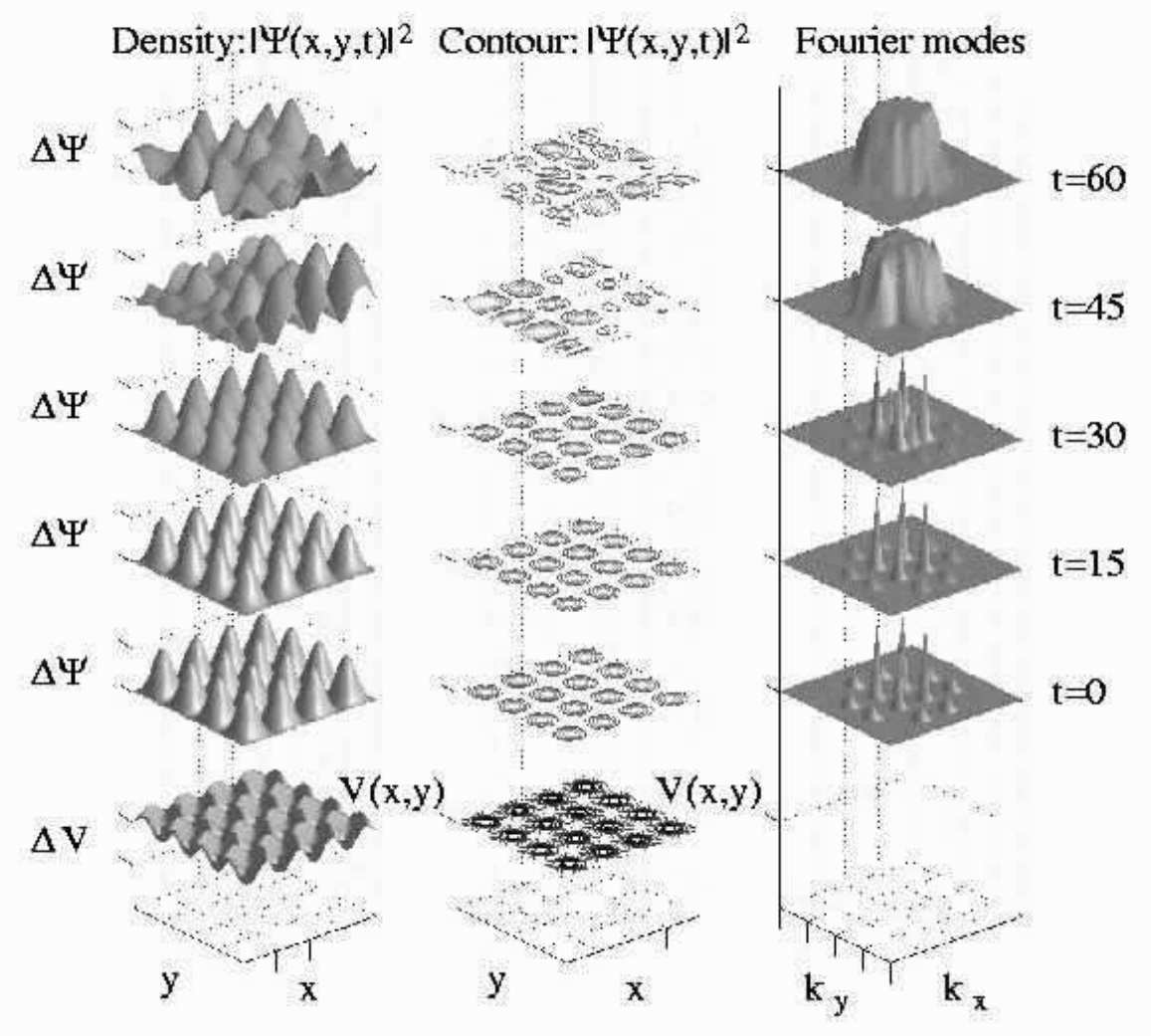

Figure 5: The two-dimensional unstable repulsive evolution of the $\operatorname{sn}\left(m_{i} x_{i}, k_{i}\right)$ solution corresponding to (16a) over four periods, with $k_{1}=k_{2}=0.5, m_{1}=m_{2}=1$, and $A_{1}=A_{2}=1$.

\subsubsection{Two-Dimensional Solutions}

The first two-dimensional solution we consider is the $\operatorname{dn}\left(m_{i} x_{i}, k_{i}\right)$ solution (160). As predicted by Theorem 1, this nodeless solution is linearly stable for a condensate with repulsive interactions. The evolution of four periods of this solution with $A_{i}=-1, m_{i}=1$ and $k=0.5, i=1,2$ for $t \in[0,60]$ is shown in Fig. 1. The three columns of this figure, and all other figures of this type, represent in order the evolution of the density $|\psi(x, y, t)|^{2}$, a contour plot of this same evolution, and the evolution of the arctan of the Fourier spectrum. The bottom picture in the first two columns shows the potential $V(x, y)$. The arctan is applied to the spectral evolution to limit the range of the power spectrum. This results in a more elucidating representation of the dynamics of the exact solution, by removing the extreme contrasts in the range of the Fourier spectrum. For the nodeless $\operatorname{dn}\left(m_{i} x_{i}, k_{i}\right)$ solution, we note that over the time range considered, and indeed for times $t \rightarrow \infty$, the solution does not change even when strongly perturbed. As argued previously [9, 10, 16], the stability of this solution is reminiscent of the stability of the plane wave solution of the nonlinear Schrödinger equation with repulsive nonlinearity [33].

In contrast to the stable $\operatorname{dn}\left(m_{i} x_{i}, k_{i}\right)$ solution, the $\operatorname{sn}\left(m_{i} x_{i}, k_{i}\right)$ and $\operatorname{cn}\left(m_{i} x_{i}, k_{i}\right)$ solutions corresponding to (16a) and (16b) are unstable. Both these solutions have nodes and violate the linear 


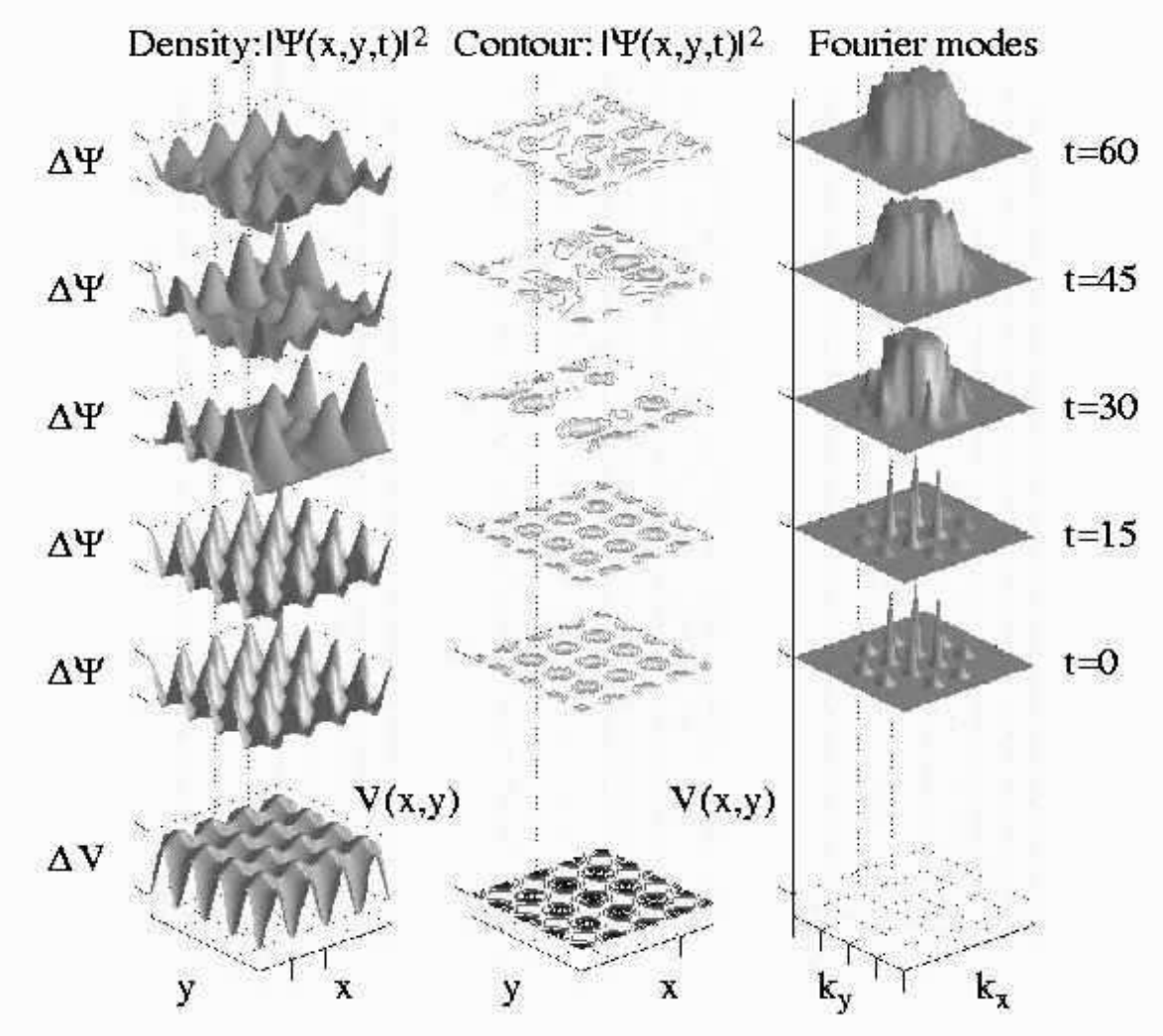

Figure 6: The two-dimensional unstable repulsive evolution of the $\operatorname{cn}\left(m_{i} x_{i}, k_{i}\right)$ solution corresponding to (16b) over four periods, with $k_{1}=k_{2}=0.5, m_{1}=m_{2}=1$, and $A_{1}=A_{2}=-1$.

stability criterion of Theorem 1. The evolution for $t \in[0,60]$ of four periods of these solutions is shown in Figs. 5 and 6 . The evolution of the density clearly shows the onset of a modulational instability which deforms the exact solution. The power spectrum shows that this modulational instability results in the activation of a large number of Fourier modes, destroying the possibility of stable evolution.

To illustrate the computational stability results, we calculate the difference $E$ between the density of the exact solution and the density of the numerical (perturbed) solution for the $\operatorname{sn}\left(m_{i} x_{i}, k_{i}\right)$, $\operatorname{cn}\left(m_{i} x_{i}, k_{i}\right)$ and $\operatorname{dn}\left(m_{i} x_{i}, k_{i}\right)$ solutions of (16a)-(160). Thus, $E=|\psi(x, y, t)|^{2}-|\psi(x, y, 0)|^{2}$. If the numerical evolution was exact and the initial condition was unperturbed by white noise, this difference would be identically zero, since the solutions we consider are stationary. Thus any growth in this difference is due to an instability mechanism which causes any errors to grow. In Fig. 7, the three columns given by $E(D n, D n), E(S n, S n)$, and $E(C n, C n)$ represent the errors in the exact solutions given by the $\operatorname{dn}\left(m_{i} x_{i}, k_{i}\right), \operatorname{sn}\left(m_{i} x_{i}, k_{i}\right)$ and $\operatorname{cn}\left(m_{i} x_{i}, k_{i}\right)$ solutions of (16a)-(160) respectively. For the $\operatorname{dn}\left(m_{i} x_{i}, k_{i}\right)$ solution, the error $E(D n, D n)$ remains at the level of the initial noise. In contrast, the errors for the $\operatorname{sn}\left(m_{i} x_{i}, k_{i}\right)$ and $\operatorname{cn}\left(m_{i} x_{i}, k_{i}\right)$ solutions, $E(S n, S n)$ and $E(C n, C n)$, start to grow at the onset of instability near $t \approx 40$ and $t \approx 20$ respectively. 


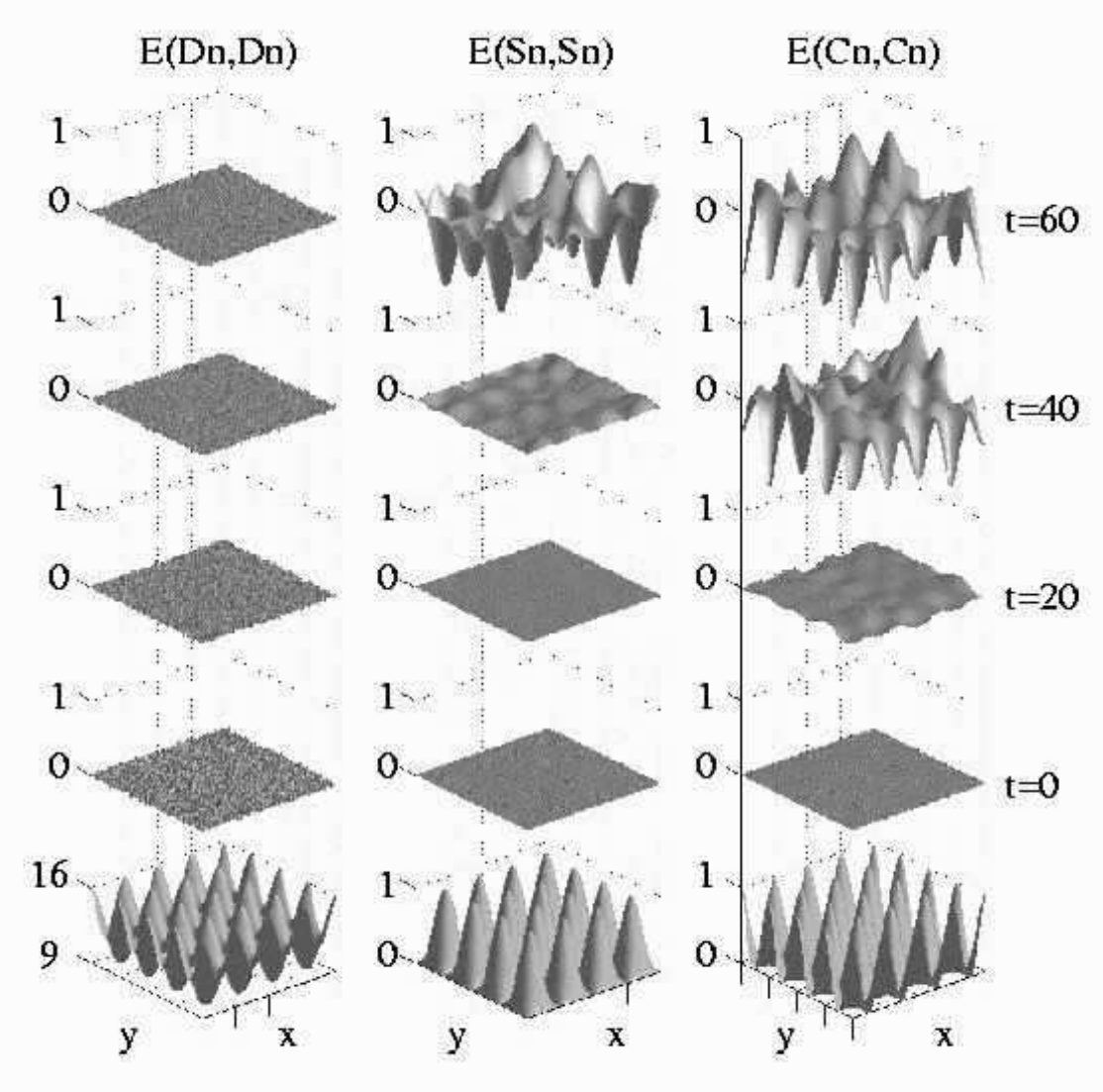

Figure 7: The two-dimensional repulsive evolution of the error, $E=|\psi(x, y, t)|^{2}-|\psi(x, y, 0)|^{2}$, for the $\operatorname{sn}\left(m_{i} x_{i}, k_{i}\right), \operatorname{cn}\left(m_{i} x_{i}, k_{i}\right)$ and $\operatorname{dn}\left(m_{i} x_{i}, k_{i}\right)$ solutions of (16a)-1160). The solutions considered correspond to Figs. 4 . The bottom row shows the respective exact solutions.

The unstable behavior is further illustrated by the evolution of the $L^{\infty}$ norm of $E$ : $\left.\max _{\{x, y\}}|| \psi(x, y, t)\right|^{2}-|\psi(x, y, 0)|^{2} \mid$. In Fig. 8, this $L^{\infty}$ norm dynamics is given for both the two-dimensional and three-dimensional solutions of the equation with repulsive nonlinearity considered numerically in this paper. The $L^{\infty}$ norm of the unstable solutions grows at the onset of the instability and saturates at a finite value. This reflects the nature of the repulsive instability, i.e., no large gradients or sharp peaks are allowed to develop in the solution. For the stable solutions, this $L^{\infty}$ norm remains at the initial noise level.

\subsubsection{Three-Dimensional Solutions}

As in the two-dimensional case, we first consider the trivial phase $\operatorname{dn}\left(m_{i} x_{i}, k_{i}\right)$ solution (16c). As shown in Theorem 1, this nodeless solution is linearly stable. The evolution of eight periods of this solution with $A_{i}=-0.5, m_{i}=1$ and $k=0.5, i=1,2,3$ for $t \in[0,40]$ is shown in Fig. 9 . The two rows of this figure, and all other figures of this type, represent the evolution of a selected density iso-surface $|\psi(x, y, z, t)|^{2}=$ constant, and the evolution of an iso-surface of the arctan of the Fourier spectrum. Unless otherwise stated, the density iso-surface shown is at $30 \%$ of the value 

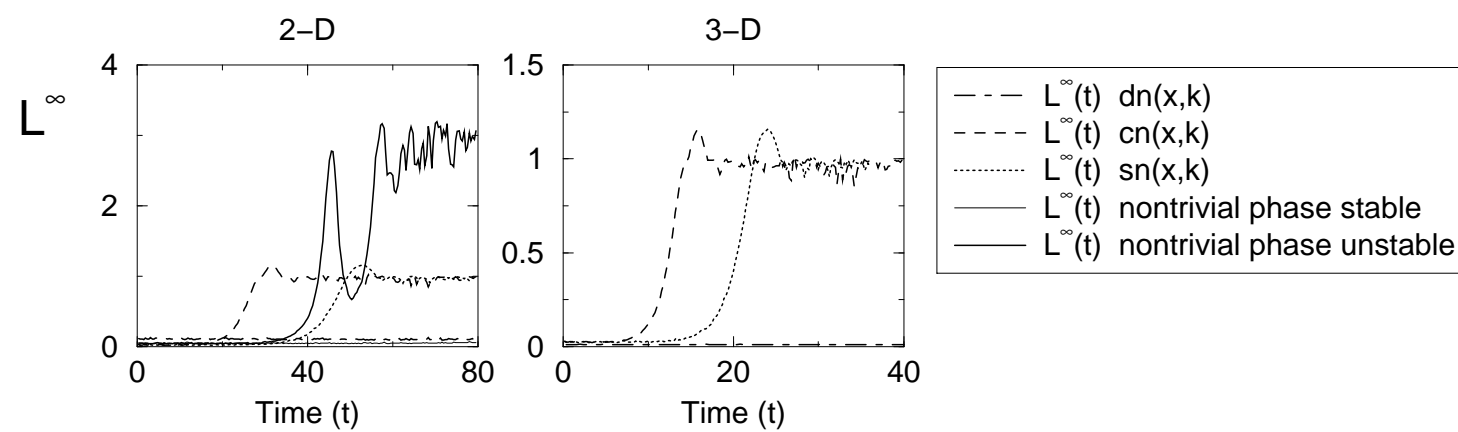

Figure 8: Evolution of the $L^{\infty}$ norm of the error in two- and three-dimensions for several trivialphase solutions of the repulsive equation.

from minimum to maximum while the iso-surface of the Fourier spectrum is at a value of one. Note that the arctan of the spectrum limits the value of the power density to a maximum of $\pi / 2$. Over the time range considered, and indeed for times $t \rightarrow \infty$, the nodeless $\operatorname{dn}\left(m_{i} x_{i}, k_{i}\right)$ solution is unaffected by the perturbations. This even holds for perturbations that are strong compared to the amplitude of the solution.

Just as in the two-dimensional case, the $\operatorname{sn}\left(m_{i} x_{i}, k_{i}\right)$ and $\operatorname{cn}\left(m_{i} x_{i}, k_{i}\right)$ solutions corresponding to (16a-b) are unstable. Both these solutions have nodes and violate the stability criterion of Theorem 1. The evolution for $t \in[0,40]$ of eight periods of these solutions is shown in Figs. 10 and 11. The evolution of the density clearly shows the onset of a modulational instability which causes break-up of the exact solution. The Fourier spectrum of the density shows that this modulational instability results in the activation of a large number of Fourier modes, destroying the possibility of stable evolution. Fig. 8 illustrates this unstable behavior by showing the evolution of the $L^{\infty}$ norm of the error: $\left.\max _{\{x, y, z\}}|| \psi(x, y, z, t)\right|^{2}-|\psi(x, y, z, 0)|^{2} \mid$. As with the two-dimensional results, the $L^{\infty}$ norm of the error of the unstable solutions grows at the onset of the instability and saturates at a finite value. For the stable solutions, this $L^{\infty}$ norm remains at the initial noise level. 

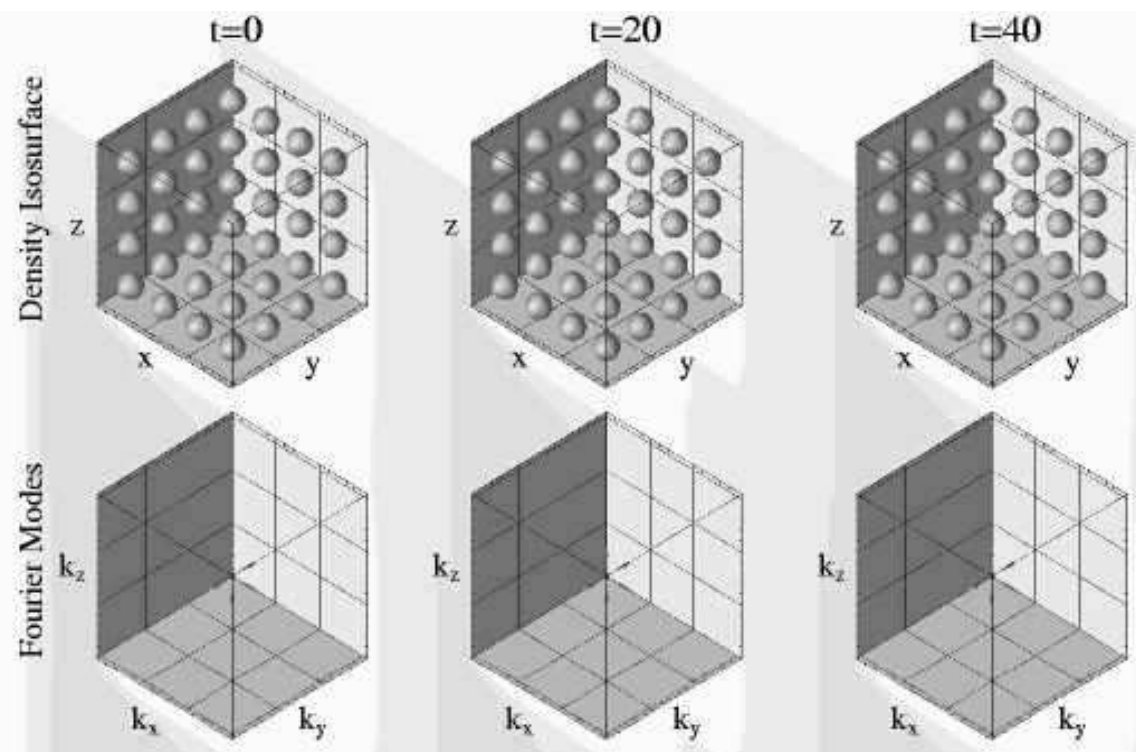

Figure 9: The three-dimensional stable repulsive evolution of the $\operatorname{dn}\left(m_{i} x_{i}, k_{i}\right)$ solution corresponding to (16c) over eight periods (two in each direction), with $k_{1}=k_{2}=k_{3}=0.5, m_{1}=m_{2}=m_{3}=1$, and $A_{1}=A_{2}=A_{3}=-0.5$. The Fourier spectrum is composed of six symmetric peaks around the origin which are obscured by the grid lines.
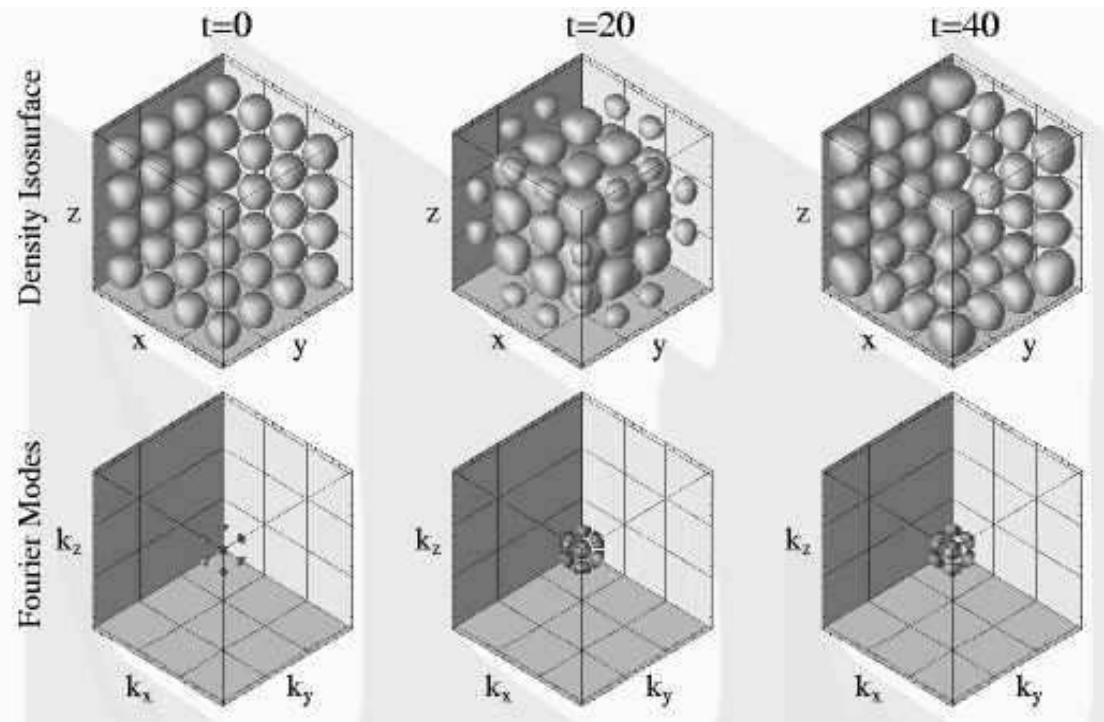

Figure 10: The three-dimensional unstable repulsive evolution of the $\operatorname{sn}\left(m_{i} x_{i}, k_{i}\right)$ solution corresponding to (16a) over eight periods (two in each direction), with $k_{1}=k_{2}=k_{3}=0.5$, $m_{1}=m_{2}=m_{3}=1$, and $A_{1}=A_{2}=A_{3}=1$. At $t=40$ the further loss of structure, which is exemplified by the Fourier spectrum, is hidden by the shape of the iso-surfaces at the boundary of the box. 

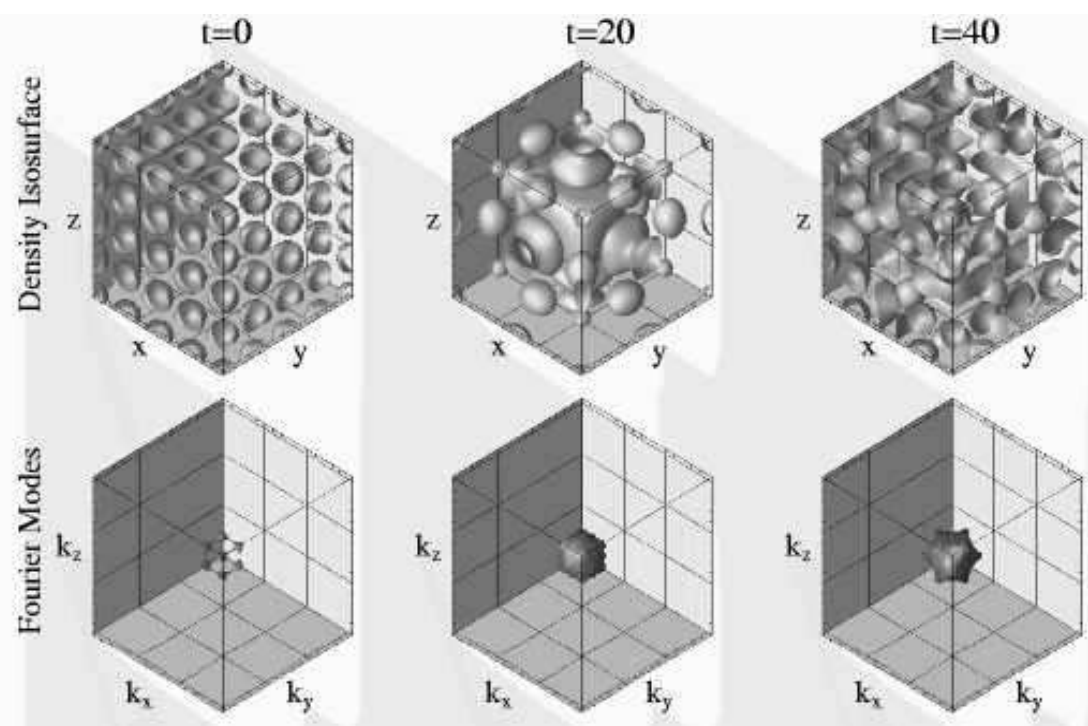

Figure 11: The three-dimensional unstable repulsive evolution of the $\operatorname{cn}\left(m_{i} x_{i}, k_{i}\right)$ solution corresponding to (16b) over eight periods (two in each direction), with $k_{1}=k_{2}=k_{3}=0.5$, $m_{1}=m_{2}=m_{3}=1$, and $A_{1}=A_{2}=A_{3}=-1$.

\subsection{Numerical Simulations: Rectangular, Irregular Lattice}

In addition to regular lattices, we can consider more complicated solutions by modifying the elliptic modulus $k_{i}$ and periodicity parameter $m_{i}$. We restrict ourselves to stable two-dimensional dynamics given by the $\operatorname{dn}\left(m_{i} x_{i}, k_{i}\right)$ solution of (16c) since they are easy to illustrate and stable solutions are the most relevant for experiments. The three-dimensional behavior follows in a straightforward manner from a generalization of these two-dimensional visualizations. We only show the stable solutions at the initial time since they are unchanged as $t \rightarrow \infty$. Figure 12 displays the various behaviors as the elliptic modulus is varied from $k=0.1$ to $k=0.999$ for various periods and values of $m_{i}$. The different stable solutions vary from periodic lattice solutions in Fig. 12a and 12k, to the well-separated and localized spikes of $12 \mathrm{~b}$, to well-separated regions of oscillation and localization 12d-f. Thus stability is independent of the lattice structure, as long as the solution is off-set from the zero level.

\subsection{Numerical Simulations: Nontrivial Phase}

Analytical results for the stability of the nontrivial phase solutions are difficult to obtain even in one dimension. Thus we rely on numerical investigations of the stability of these solutions. To avoid the complications which arise from phase quantization, consider the solutions (17) that are trigonometric in all directions and for which phase quantization is automatically satisfied. For the NLS equation with repulsive nonlinearity (11) in two- and three-dimensions, these solutions are stable or unstable depending on the offset parameters $B_{1}$ and $B_{2}$ (two-dimensions) or $B_{1}, B_{2}$ and $B_{3}$ (three-dimensions). As shown in Fig. 13 the off-set solution in two-dimensions, which is qualitatively like the stable $\operatorname{dn}\left(m_{i} x_{i}, k_{i}\right)$ solution, is stable with $B_{1}=1$ and $B_{2}=0.7$ whereas in Fig. 14 the unstable nontrivial phase solution which is below the offset threshold is illustrated with 
(a)

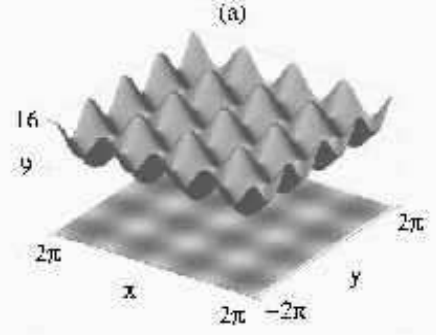

(d)

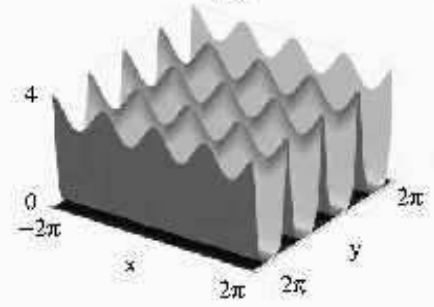

(b)

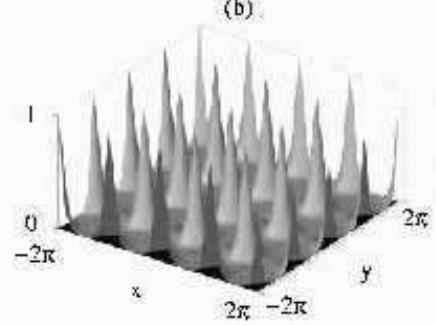

(c)

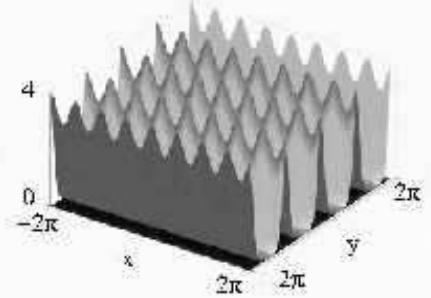

(e)

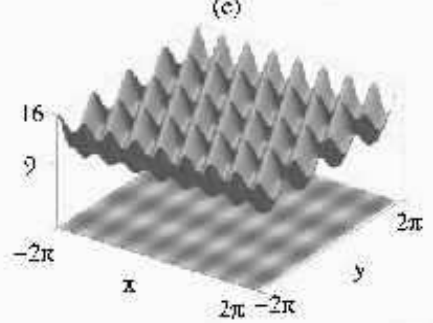

(1)

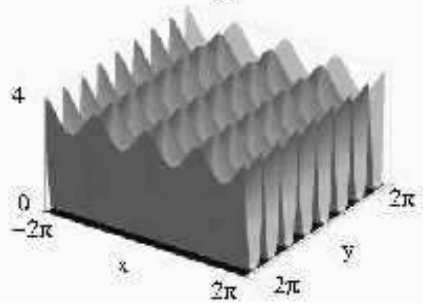

Figure 12: Stable two-dimensional solutions with irregular lattice potentials and $m_{i}$ values chosen so that the solutions are $4 \pi$-periodic. (a) Two periods in each direction, with $k_{1}=0.1$ and $k_{2}=0.1$; (b) two periods in each direction, with $k_{1}=0.9$ and $k_{2}=0.9$; (c) four periods in $x$ and two periods in $y$, with $k_{1}=0.1$ and $k_{2}=0.1$ respectively; (d) two periods in each direction, with $k_{1}=0.1$ and $k_{2}=0.9$; (e) four periods in $x$ and two periods in $y$, with $k_{1}=0.1$ and $k_{2}=0.9$ respectively; (f) two periods in $x$ and four periods in $y$, with $k_{1}=0.1$ and $k_{2}=0.9$ respectively. The spatial domains are normalized to $x, y \in[-2 \pi, 2 \pi]$.

$B_{1}=1$ and $B_{2}=0.6$. These nontrivial phase solutions illustrate the necessity of offset for stability. In particular, for the values considered, an offset threshold is achieved at $B_{2} \approx 0.65$.

Similar results for repulsive nontrivial phase solutions hold in three dimensions: the offset parameter $B_{i}$ is the crucial parameter which determines the stability of a given nontrivial phase solution. Figure 15 shows the stability of an off-set solution in three-dimensions with $B_{1}=B_{2}=$ $B_{3}=1$ whereas in Fig. 16 an unstable nontrivial phase solution, which is below the instability threshold, is illustrated with $B_{1}=B_{2}=B_{3}=0.5$.

Mixed solutions can also be considered: these are solutions with nontrivial phase (17) in one or more directions and trivial phase (16a) in the remaining directions. As before, offset determines the stability of these solutions. To illustrate this, we consider the two-dimensional repulsive evolution with a trivial phase $\operatorname{dn}\left(m_{i} x_{i}, k_{i}\right)$ solution of (16c) in the $y$ direction and a nontrivial phase solution (17) in the $x$ direction. The $\operatorname{dn}\left(m_{i} x_{i}, k_{i}\right)$ solution of (160) was found to be stable whereas the nontrivial phase solution (17) was stable provided a sufficient offset was present. Following the previous paragraphs, we shown in Fig. 17 the mixed solution with offset parameter $B_{1}=0.4$ for the nontrivial phase dimension. The resulting evolution is stable under perturbation as $t \rightarrow \infty$. Alternatively, when the offset parameter is decreased to $B_{1}=0.3$, the nontrivial phase solution is unstable, as observed in Fig. 18. 


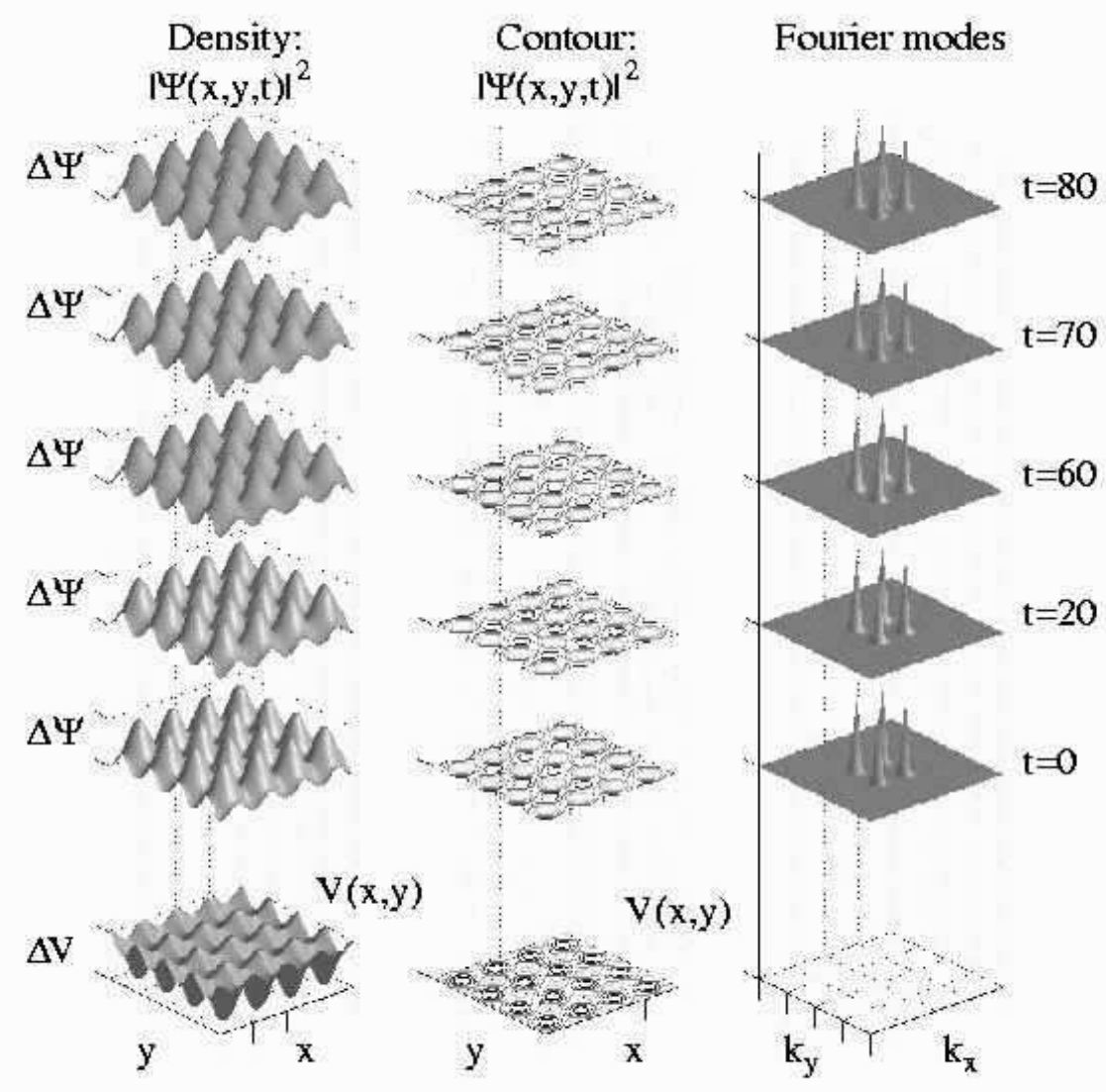

Figure 13: The two-dimensional stable defocusing evolution of the nontrivial phase solution (117) with four periods, and $k_{1}=k_{2}=0.0, m_{1}=m_{2}=1, A_{1}=A_{2}=1, B_{1}=1$, and $B_{2}=0.7$.

\section{Condensates with attractive interaction}

In this section, the numerical solutions in the attractive regime of the nonlinear Schrödinger equation (11) are considered. The initial conditions are only selected from the exact trivial-phase solutions given by (16a-c). These trivial phase solutions, given by the $\operatorname{sn}\left(m_{i} x_{i}, k_{i}\right), \operatorname{cn}\left(m_{i} x_{i}, k_{i}\right)$ and $\operatorname{dn}\left(m_{i} x_{i}, k_{i}\right)(i=1,2$, or 3$)$ of $(16 \mathrm{a}-\mathrm{c})$, characterize the basic solution types: peak-on-peak with nodes, peak-on-peak without nodes, peak-on-trough with nodes, and peak-on-trough without nodes.

It is well known [33] that in two dimensions and higher, solutions of the attractive nonlinear Schrödinger equation undergo a process of collapse and blow-up. This process is also possible in the presence of a periodic potential. It is briefly discussed in Appendix B. In addition, plane wave solutions are known to be modulationally unstable [33]. Thus we do not expect stable two- and three-dimensional solutions. However, it may be possible to obtain a solution for which the onset of instability occurs on a longer time scale than the experimental lifetime of a condensate. This possibility is examined numerically. 


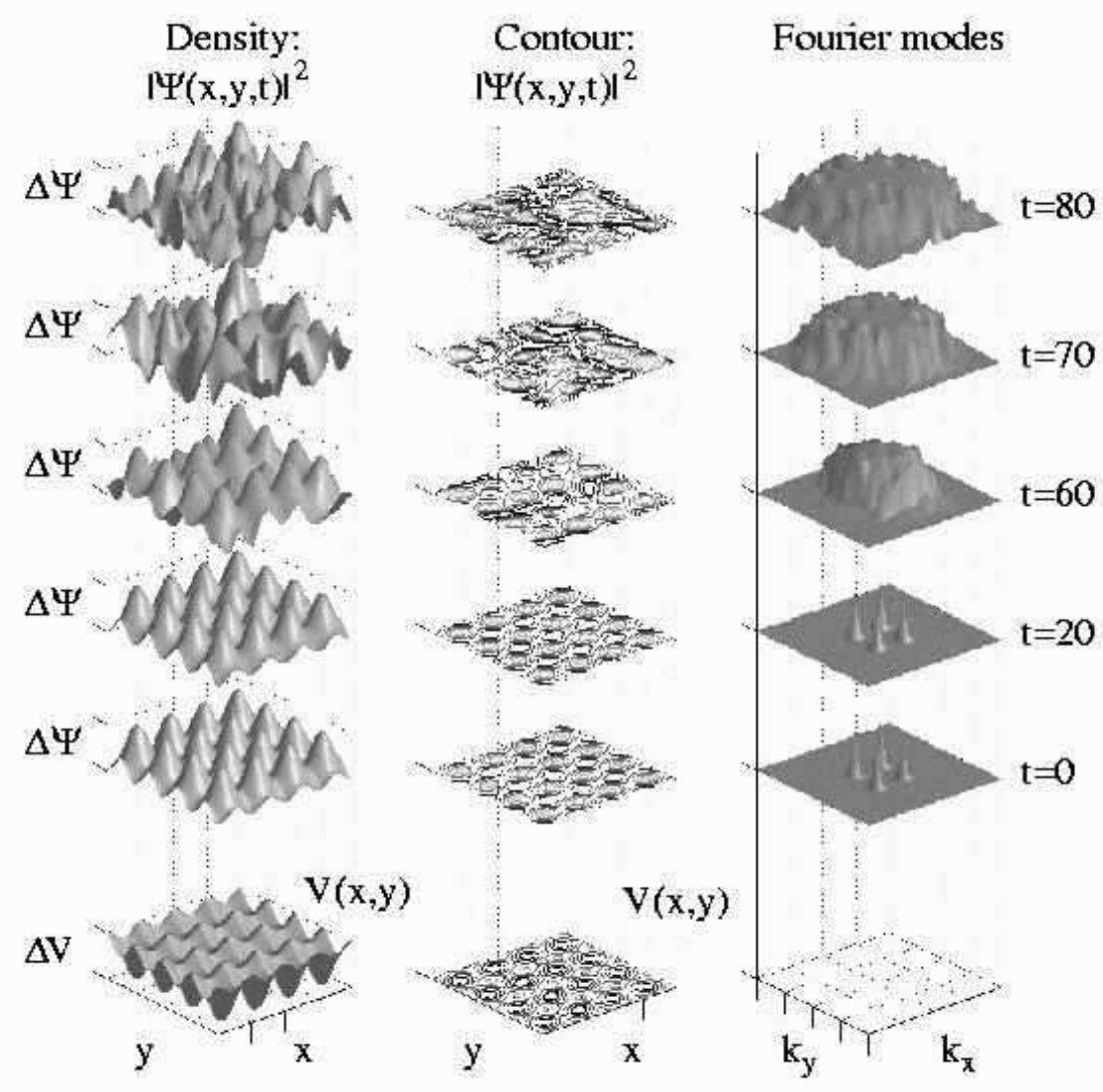

Figure 14: The two-dimensional unstable repulsive evolution of the nontrivial phase solution (17) with four periods, with $k_{1}=k_{2}=0.0, m_{1}=m_{2}=1, A_{1}=A_{2}=1, B_{1}=1$, and $B_{2}=0.6$.

\subsection{Numerical Simulations: Square, Regular Lattice}

Our numerical considerations begin with the case of a square, regular lattice. Thus the spatial domains of all $x_{i}$ are identical as are the elliptic moduli $k_{i}$. Thus, the generated lattice potential $V(\vec{x})$ and the solution $\psi(\vec{x}, t)$ do not distinguish between the different directions $x_{i}$. 

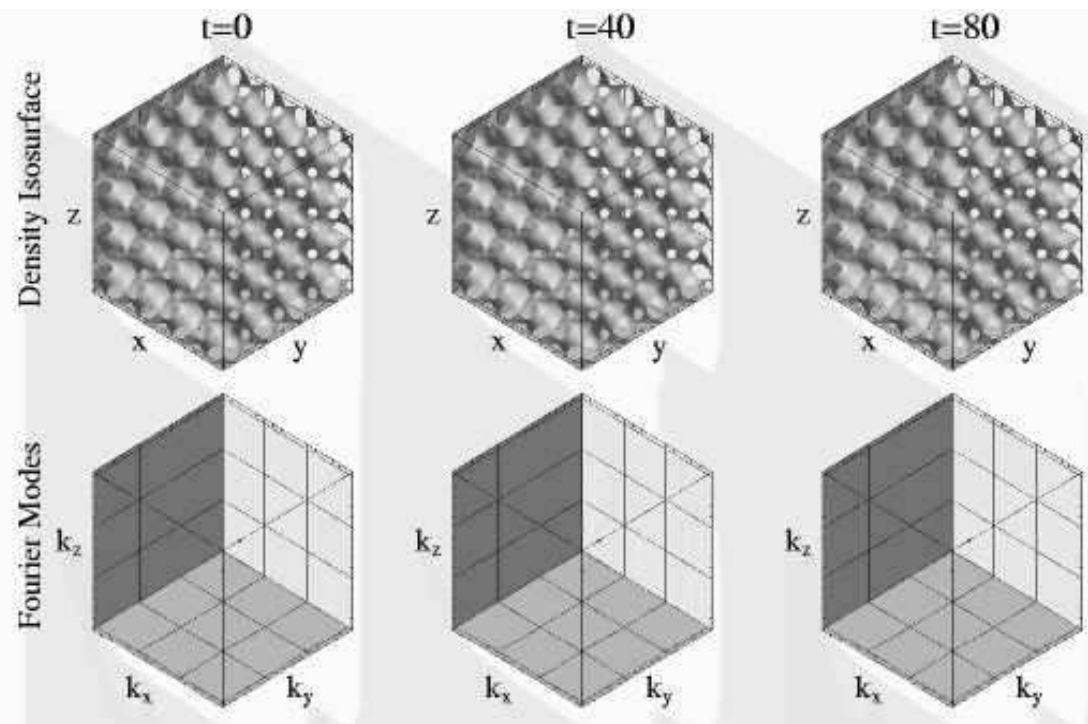

Figure 15: The three-dimensional stable repulsive evolution of the nontrivial phase solution (17) with eight periods (two in each direction), and $k_{1}=k_{2}=k_{3}=0.0, m_{1}=m_{2}=m_{3}=1$, $A_{1}=A_{2}=A_{3}=1$, and $B_{1}=B_{2}=B_{3}=1$. The Fourier spectrum is composed of six symmetric peaks around the origin which are obscured by the grid lines.

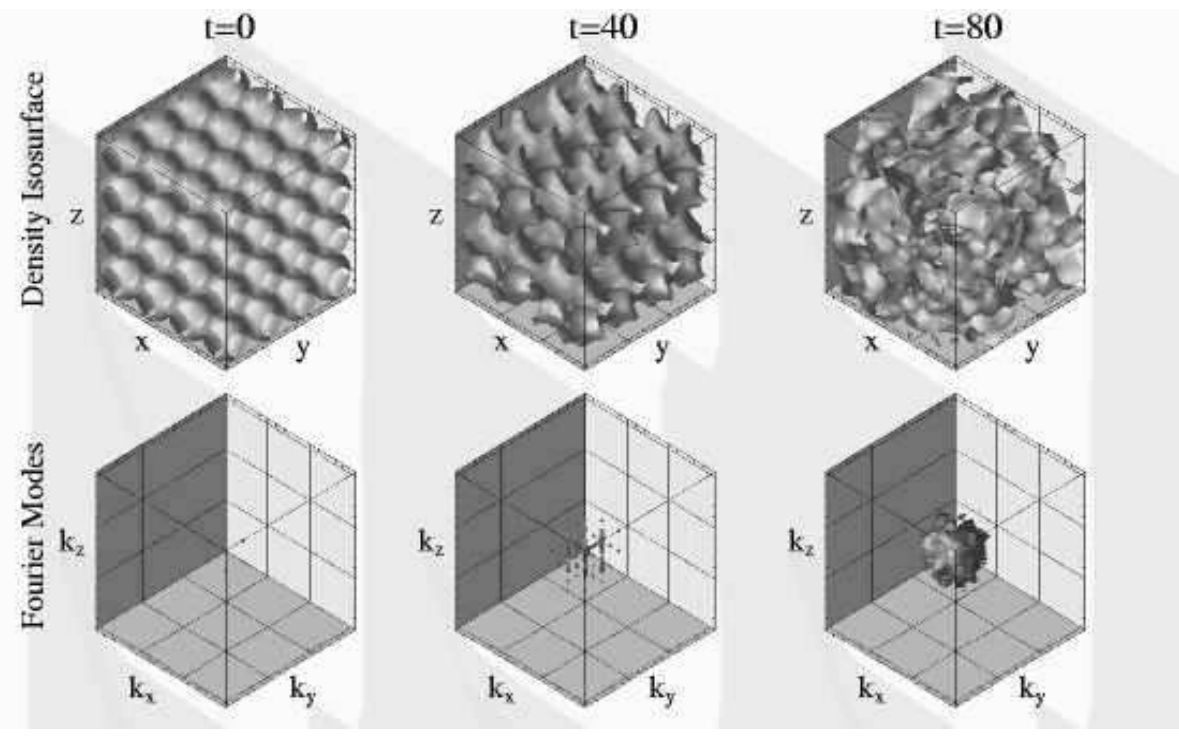

Figure 16: The three-dimensional unstable repulsive evolution of the nontrivial phase solution (17) with eight periods (two in each direction), with $k_{1}=k_{2}=k_{3}=0.0, m_{1}=m_{2}=m_{3}=1$, $A_{1}=A_{2}=A_{3}=1$, and $B_{1}=B_{2}=B_{3}=0.5$. 


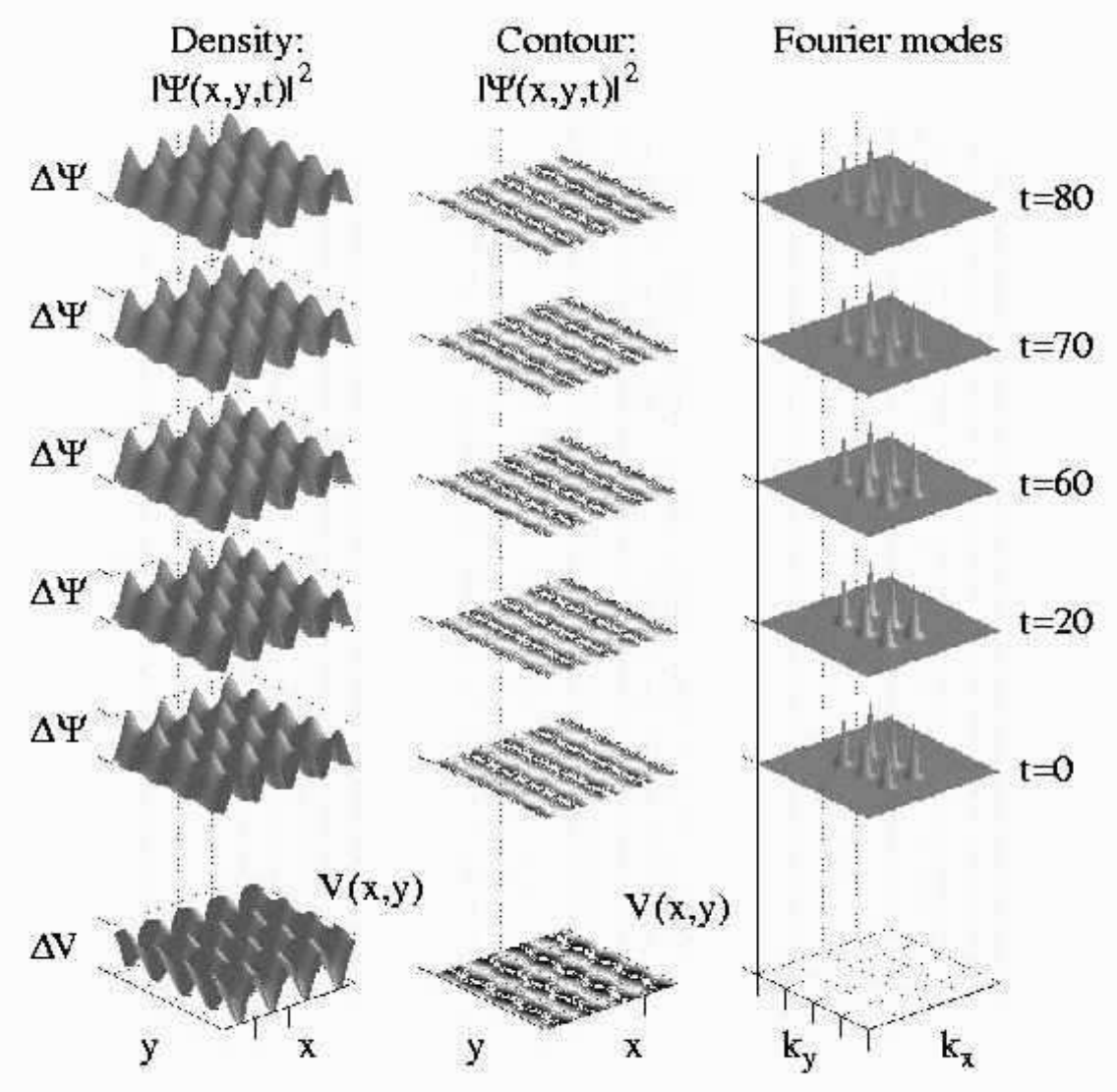

Figure 17: The two-dimensional stable repulsive evolution of a mixed solution which has nontrivial phase profile (17) in the $x$ direction and the $\operatorname{dn}\left(m_{i} x_{i}, k_{i}\right)$ profile of (160) in the $y$ direction. The figure shows two periods in each direction with $k_{1}=0, k_{2}=0.5, m_{1}=m_{2}=1, A_{1}=1, A_{2}=-0.5$, and $B_{1}=0.4$.

\subsubsection{Two-Dimensional Solutions}

The first two-dimensional solution considered is the $\operatorname{dn}\left(m_{i} x_{i}, k_{i}\right)$ solution (16c). For the attractive nonlinear Schrödinger equation, the $\operatorname{dn}\left(m_{i} x_{i}, k_{i}\right)$ solution is proved to be unstable in Theorem 2. This is a manifestation of the modulational instability of plane waves [33]. Although the modulational instability is present in the dynamics, the behavior is dominated by the collapse and blow-up phenomena which are characteristic of the attractive regime. This behavior is observed in Fig. 19 . In this case, $\ln \left(1+|\psi(x, y, t)|^{2}\right)$, and its contours are plotted to better illustrate the collapse and blow-up. The evolution of four periods of this solution with $A_{i}=-1, m_{i}=1$ and $k=0.5, i=1,2$ for $t \in[0,0.56]$ shows how quickly this phenomenon occurs.

In order to effectively capture the onset of collapse, we have aided the instability in some simulations by considering initial conditions which have twice the amplitude of an exact solution. Our motivation in expediting the instability is to limit computational cost: since the exact solutions are stationary, sufficient perturbation is required to achieve collapse. Building up such perturbations from our regular white-noise perturbations requires long time scales relative to the time scale on which collapse occurs (on the order of 1000 times longer for our numerical experiments). In order 


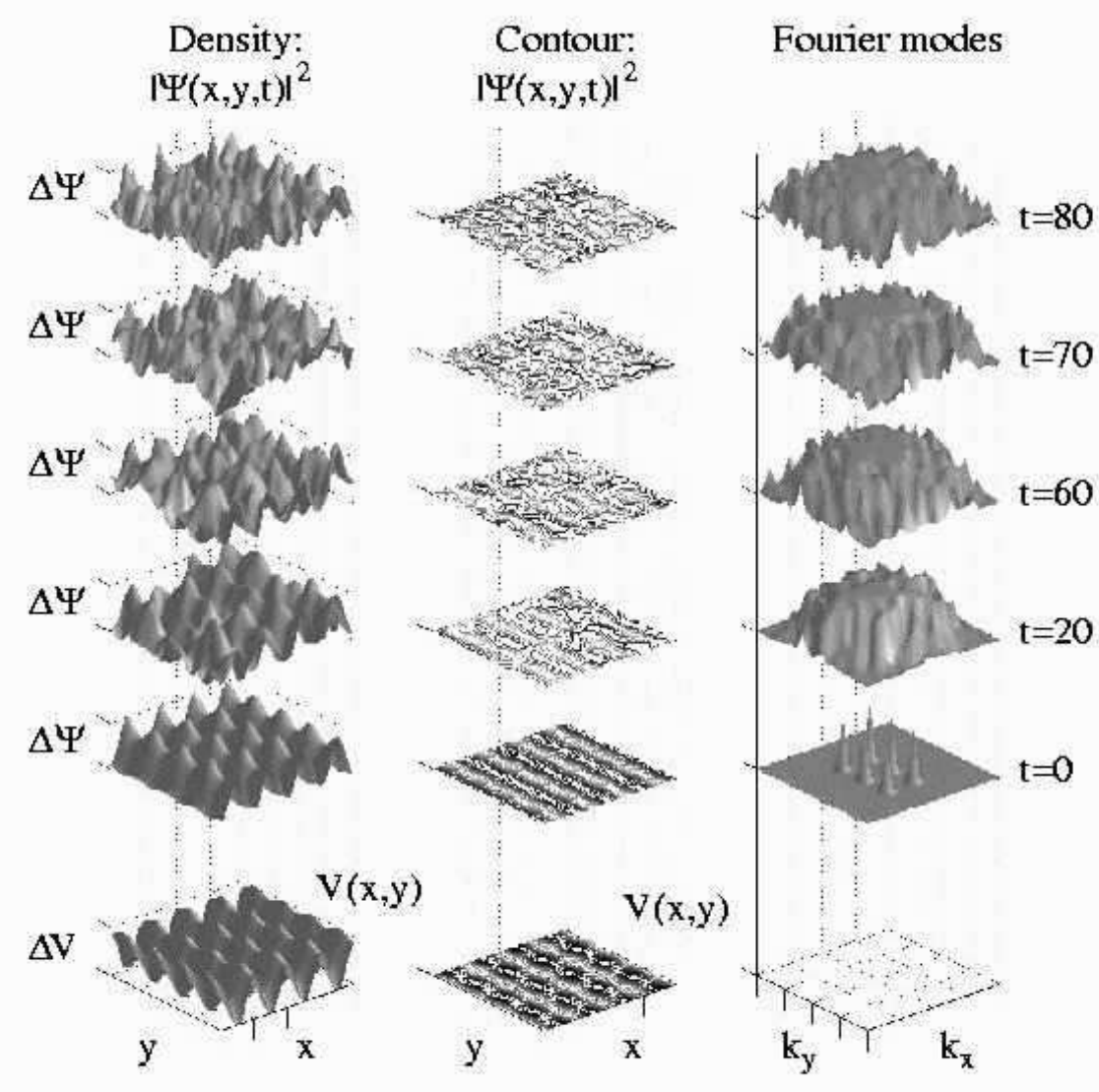

Figure 18: The two-dimensional unstable repulsive evolution of a mixed solution which has a nontrivial phase profile (17) in the $x$ direction and the $\operatorname{dn}\left(m_{i} x_{i}, k_{i}\right)$ profile of $(16 \mathrm{c})$ in the $y$ direction. The figure shows two periods with $k_{1}=0, k_{2}=0.5, m_{1}=m_{2}=1, A_{1}=1, A_{2}=-0.5$, and $B_{1}=0.3$.

to resolve the collapse, a small time step is required, which is unnecessary for the time leading up to collapse. An alternative approach to avoid this problem is to use an adaptive time-stepping algorithm. The main effect of our approach is to alter the time of collapse and blow-up. Other qualitative features of the solution are unaffected.

Note that the double-amplitude $\operatorname{dn}\left(m_{i} x_{i}, k_{i}\right)$ initial condition, which is nodeless and has a larger $L^{2}$ norm than either the $\operatorname{sn}\left(m_{i} x_{i}, k_{i}\right)$ or $\operatorname{cn}\left(m_{i} x_{i}, k_{i}\right)$ solution, collapses into a few distinct peaks. Additionally, the spreading of the Fourier mode spectrum reflects the nature of the localization inherent to collapse.

The exact peak-on-peak $\operatorname{sn}\left(m_{i} x_{i}, k_{i}\right)$ solution is also unstable for attractive condensates as shown in Fig. 20. As with the $\operatorname{dn}\left(m_{i} x_{i}, k_{i}\right)$ initial condition, the solution eventually blows up near $t \approx 31$. In this case, the total $L^{2}$ norm is much smaller than that of the $\operatorname{dn}\left(m_{i} x_{i}, k_{i}\right)$ solution so that only a single collapse peak is observed. In contrast to Fig. 19, the initial conditions are the exact solutions with a small amount of white noise added.

The $\operatorname{cn}\left(m_{i} x_{i}, k_{i}\right)$ solution can be either peak-on-peak or peak-on-trough, depending on the parameters. In the absence of the mean-field nonlinearity, the peak-on-trough $\operatorname{cn}\left(m_{i} x_{i}, k_{i}\right)$ solution 


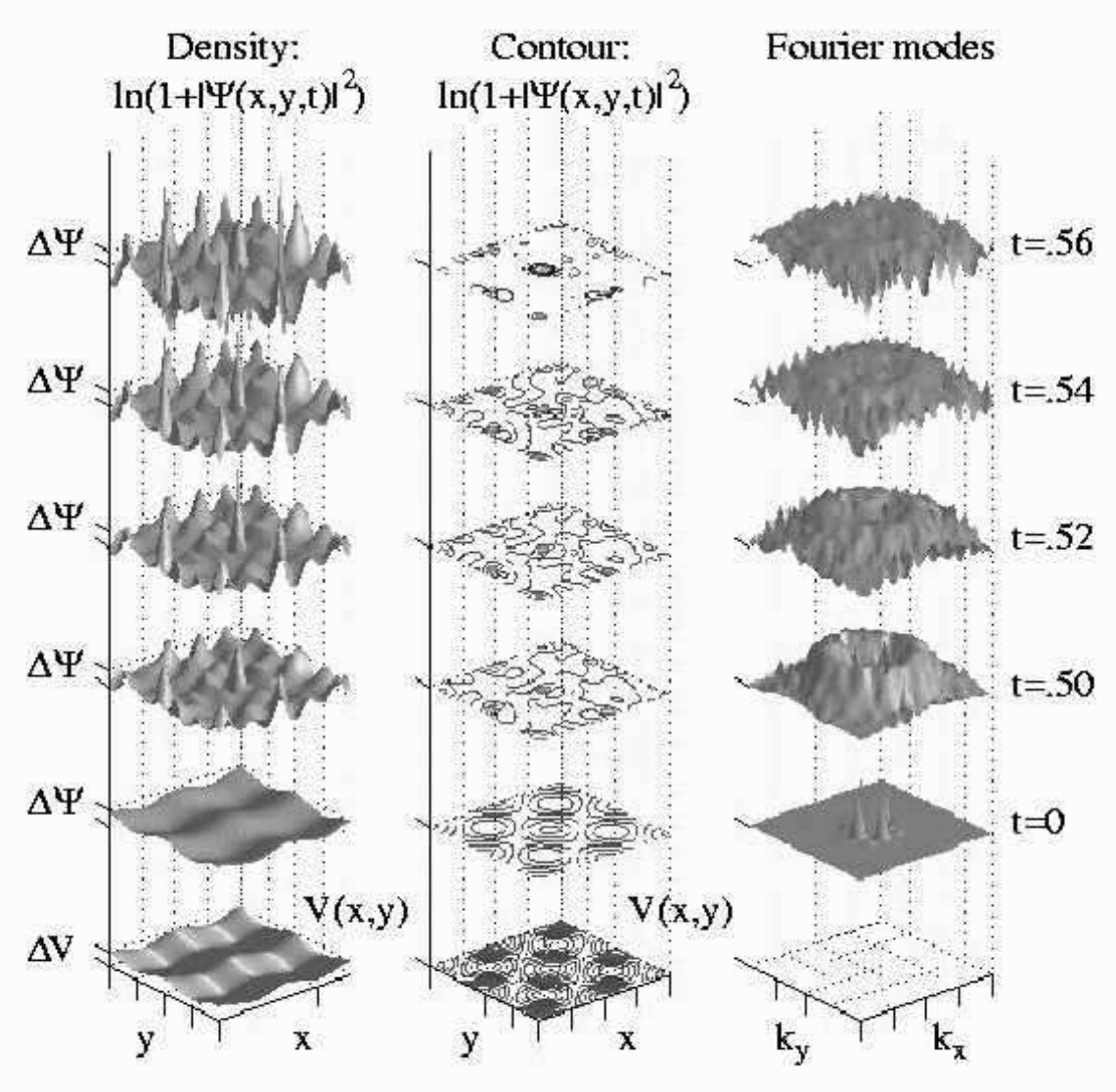

Figure 19: The two-dimensional unstable attractive evolution of the double-amplitude $\operatorname{dn}\left(m_{i} x_{i}, k_{i}\right)$ initial condition corresponding to (160) over four periods, with $k_{1}=k_{2}=0.5, m_{1}=m_{2}=1$, and $A_{1}=A_{2}=-1$.

would be stable. However, the cubic nonlinearity once again gives rise to collapse and blow-up. The evolution of a double-amplitude initial condition is shown in Fig. 21 with $A_{1}=A_{2}=-1$. The collapse of this evolved initial condition occurs shortly after $t \approx 0.47$, with the formation of well-defined growing peaks at the locations of the maxima of the initial condition. Since it is the nonlinearity which drives the collapse, we inhibit the blow-up by choosing an exact solution with a lower amplitude and smaller resulting nonlinearity. With $A_{1}=A_{2}=-0.5$, the dynamics of Fig. 22 is significantly different from that of Fig. 21. In particular, the onset of collapse does not occur until $t \approx 200$. Furthermore, the $L^{2}$ norm for the 16 periods considered computationally is just large enough to lead to collapse. Thus for small-amplitude solutions, it may be possible to obtain a periodic condensate in the lattice potential over the lifetime of the experiment. Note from Table 1, that $\delta t=200$ far exceeds the time scale of any experiment with attractive condensates.

The unstable behavior is further illustrated by the evolution of the $L^{\infty}$ norm of the error: $\left.\max _{\{x, y\}}|| \psi(x, y, t)\right|^{2}-|\psi(x, y, 0)|^{2} \mid$. In Fig. 23, the dynamics of the $L^{\infty}$ norm is given for both the two-dimensional and three-dimensional solutions of the attractive equation considered numerically in this paper. The $L^{\infty}$ norm of the unstable attractive solutions grow to infinity at the onset of the collapse of the peaks. This illustrates the fundamental nature of the attractive collapse and 


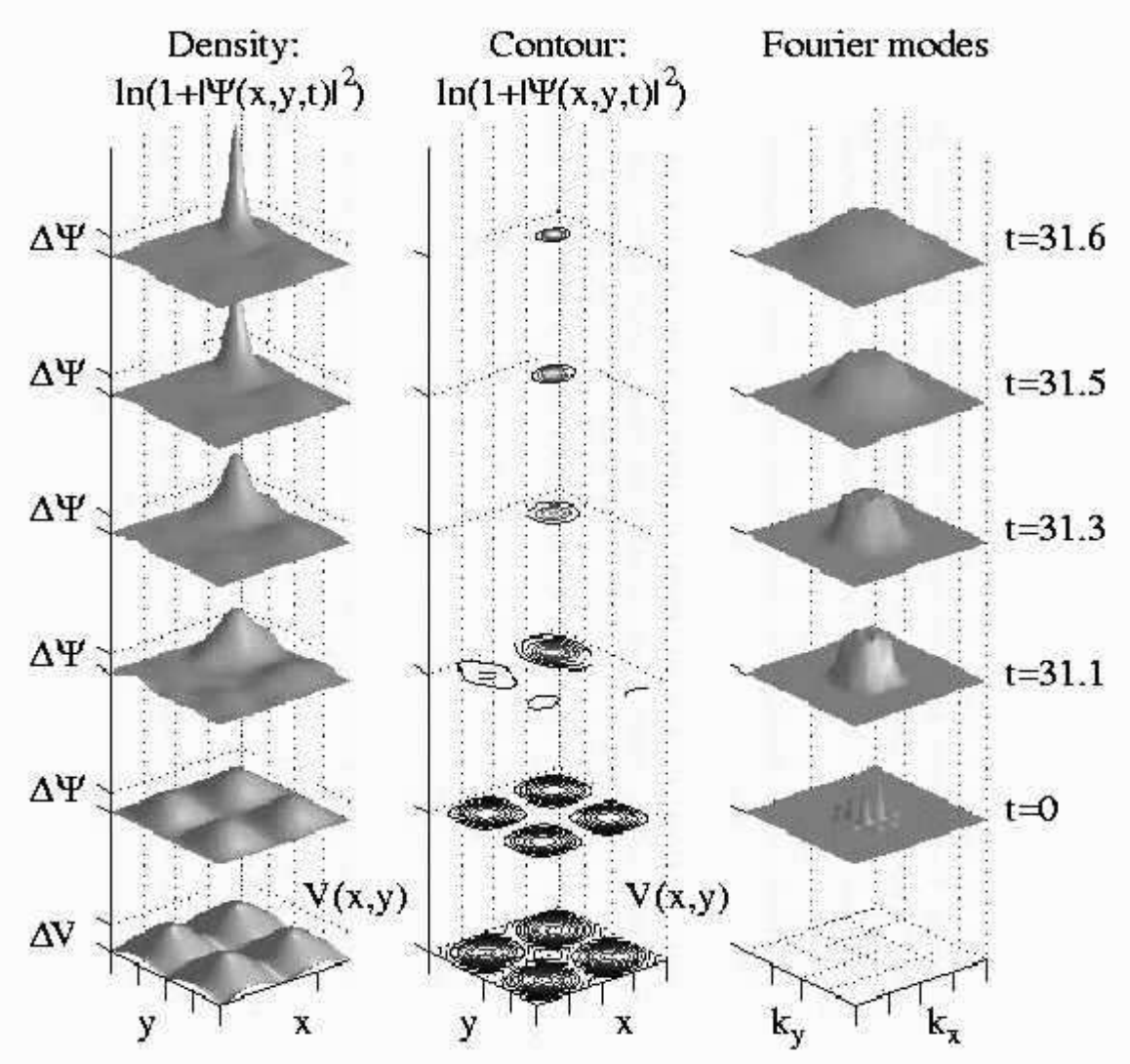

Figure 20: The two-dimensional unstable attractive evolution of the $\operatorname{sn}\left(m_{i} x_{i}, k_{i}\right)$ solution corresponding to (16c) over four periods, with $k_{1}=k_{2}=0.5, m_{1}=m_{2}=1$, and $A_{1}=A_{2}=1$.

blow-up, i.e., large gradients or sharp peaks develop in the solution.

\subsubsection{Three-Dimensional Solutions}

Following the discussion of the two-dimensional solutions, we consider the trivial phase $\operatorname{dn}\left(m_{i} x_{i}, k_{i}\right)$ solution (16c). Also in three dimensions the plane-wave solution is modulationally unstable and the solution is linearly unstable. The unstable behavior however is dominated by collapse and blow-up which are characteristic of the attractive regime. The evolution of two periods of this solution with $A_{i}=-0.25, m_{i}=1$ and $k=0.5, i=1,2,3$ for $t \in[0,0.114]$ is shown in Fig. 24. As with the two-dimensional solutions, we expedite the collapse and blow-up by doubling the amplitude of some of our exact solutions. The three rows of this figure, and of all other figures of this type, represent a slice in the $(x, y)$-plane of the evolution of the condensate density $|\psi(x, y, 0, t)|^{2}$, an iso-surface of $|\psi(x, y, z, t)|^{2}$, and the evolution of an iso-surface of the arctan of the Fourier spectrum. For the attractive equation, the displayed iso-surface for the density is at $50 \%$ of the value from minimum to maximum while the iso-surface of the Fourier spectrum is shown at a value of one. All other 


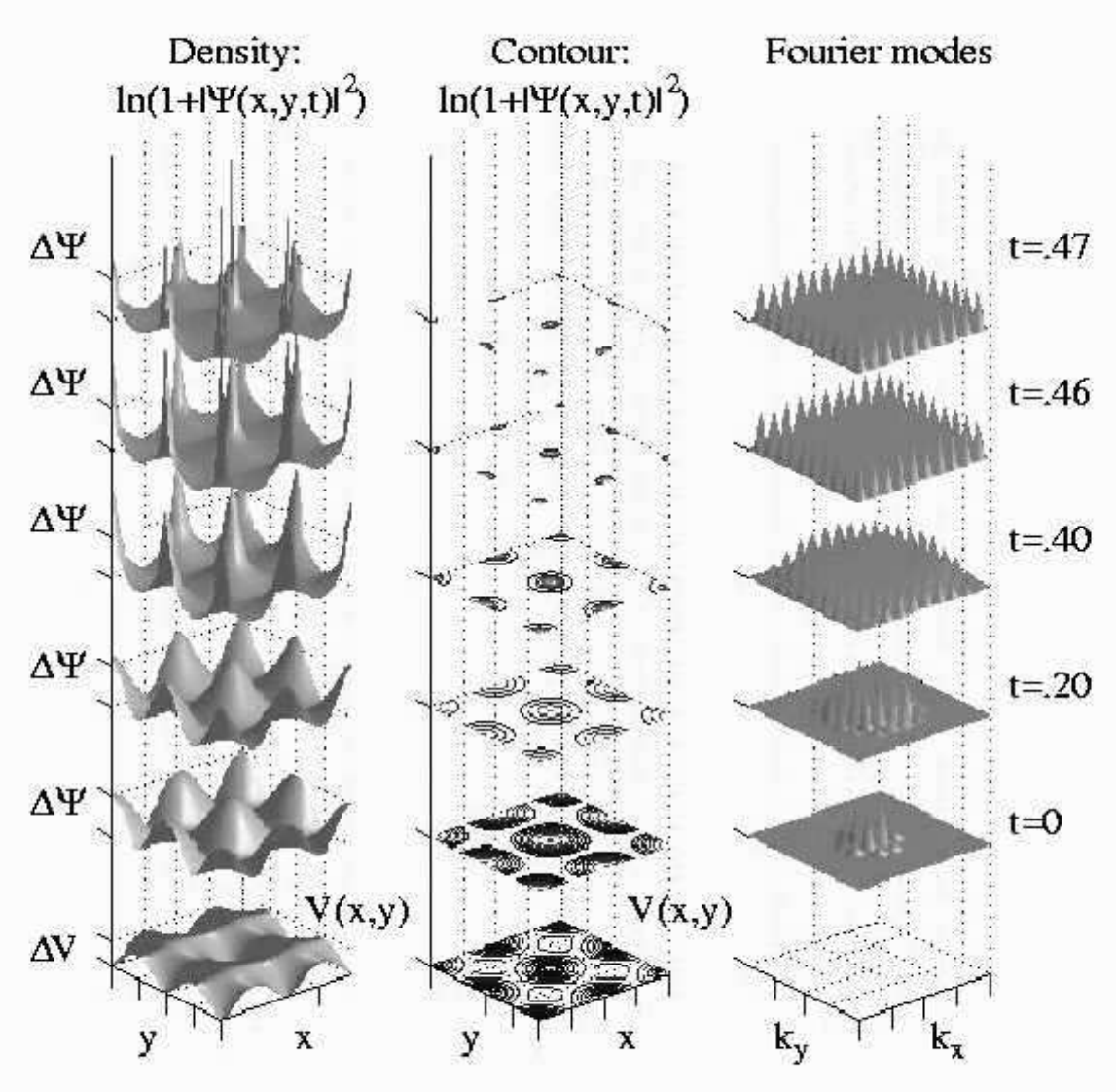

Figure 21: The two-dimensional unstable attractive evolution of the double-amplitude $\operatorname{cn}\left(m_{i} x_{i}, k_{i}\right)$ initial condition corresponding to (160) with four periods, and $k_{1}=k_{2}=0.5, m_{1}=m_{2}=1$, and $A_{1}=A_{2}=-1$. 


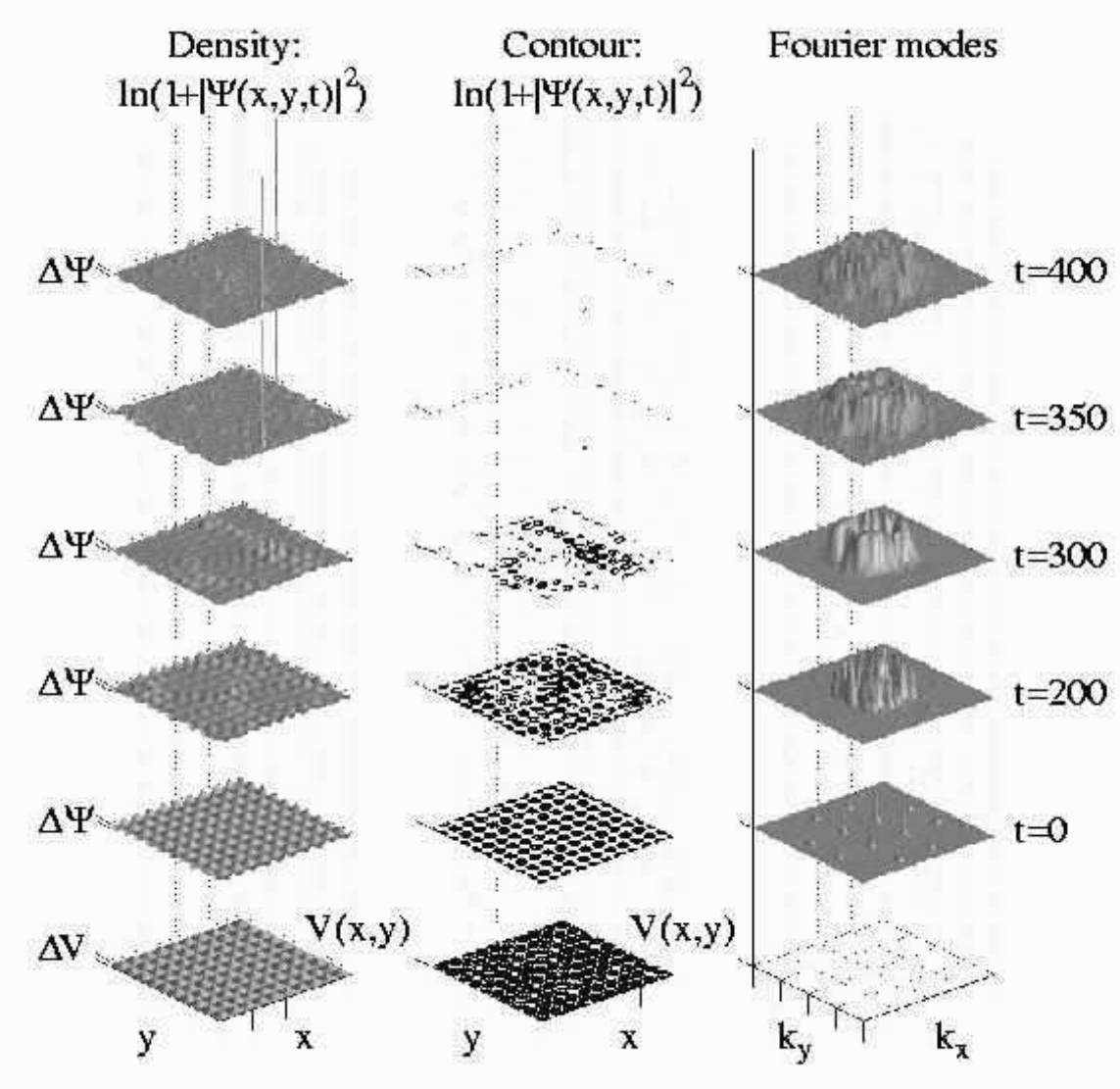

Figure 22: The two-dimensional unstable attractive evolution of the exact $\mathrm{cn}\left(m_{i} x_{i}, k_{i}\right)$ solution corresponding to (160) with 16 periods, and $k_{1}=k_{2}=0.5, m_{1}=m_{2}=1$, and $A_{1}=A_{2}=-0.5$. Note that the collapse time is well beyond $t \approx 200$.
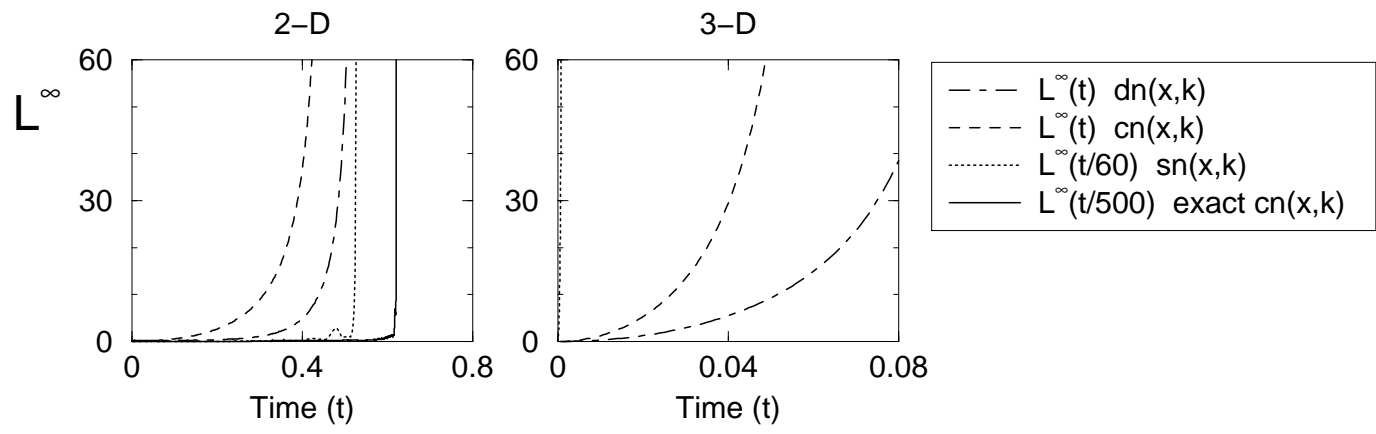

Figure 23: Evolution of the $L^{\infty}$ norm of the error in two- and three-dimensions for several trivialphase solutions of the attractive equation. 

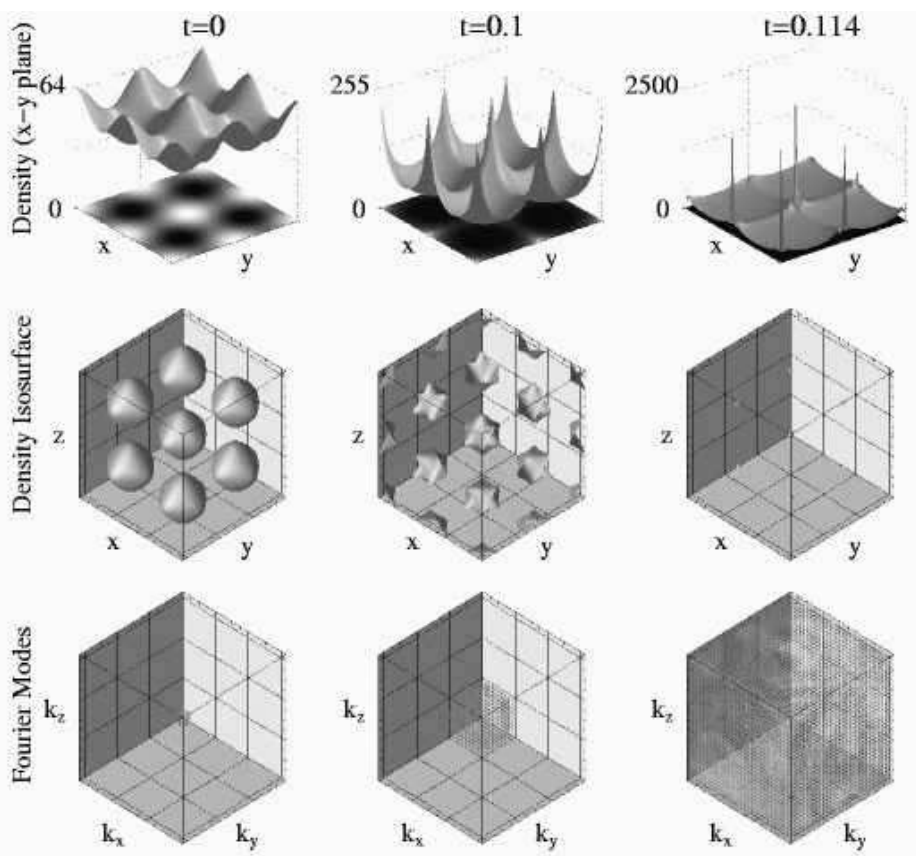

Figure 24: The three-dimensional unstable attractive evolution of the double-amplitude $\operatorname{dn}\left(m_{i} x_{i}, k_{i}\right)$ initial condition corresponding to (16c) with eight periods (two in each direction), with $k_{1}=k_{2}=k_{3}=0.5, m_{1}=m_{2}=m_{3}=1$, and $A_{1}=A_{2}=A_{3}=-0.25$.
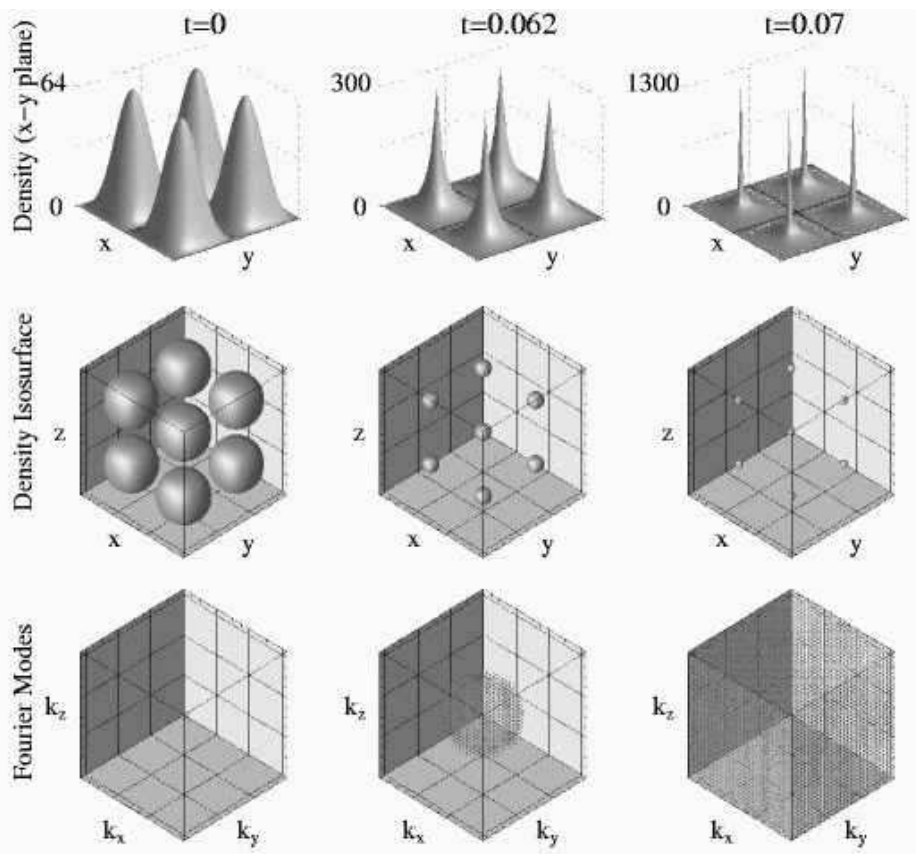

Figure 25: The three-dimensional unstable attractive evolution of a double-amplitude $\operatorname{sn}\left(m_{i} x_{i}, k_{i}\right)$ initial condition corresponding to (16c) with eight periods (two in each direction), with $k_{1}=k_{2}=$ $k_{3}=0.5, m_{1}=m_{2}=m_{3}=1$, and $A_{1}=A_{2}=A_{3}=1$. 
figures of this type are similar. This figure clearly shows the localization of the collapsing solution which results in the spreading of the Fourier modes in the spectrum.

A double-amplitude $\operatorname{sn}\left(m_{i} x_{i}, k_{i}\right)$ peak-on-peak initial condition is also unstable in the attractive limit as shown in Fig. 25. As with the $\operatorname{dn}\left(m_{i} x_{i}, k_{i}\right)$ initial condition, the dynamics eventually lead to blow-up near $t \approx 0.07$. In this case, the total $L^{2}$ norm is much larger than for its two-dimensional counterpart so that more distinct peaks are observed during the collapse process.

As in two dimensions, an exact peak-on-trough $\operatorname{cn}\left(m_{i} x_{i}, k_{i}\right)$ solution is stable in the absence of the mean-field nonlinearity. However, the cubic nonlinearity once again gives rise to collapse and blow-up. The evolution of a double-amplitude initial condition is shown in Fig. 26 with $A_{1}=A_{2}=-1$. The dynamics leads to collapse of this initial condition at $t \approx 0.07$, and the formation of a few well-defined peaks is observed. Since it is the nonlinearity which drives the collapse phenomena, we inhibit the blow-up by choosing an exact initial condition with lower amplitude and smaller resulting nonlinearity. Choosing $A_{1}=A_{2}=-0.5$, the resulting dynamics of Fig. 27 is shown to be significantly different from those considered previously. In particular, the onset of collapse is arrested by the fact that only two periods in each direction are considered so that the $L^{2}$ norm is small, and the solution remains in the linear regime for $t \in[0,2000]$. This again suggests the possibility of obtaining a stable periodic condensate in a lattice potential over the lifetime of an experiment.

\section{Discussion}

We considered the repulsive and attractive nonlinear Schrödinger equation with an elliptic function potential in two and three dimensions. Periodic solutions of this equation were constructed and their dynamical stability was investigated analytically and numerically. For condensates with repulsive atomic interactions, all stable, trivial-phase solutions are deformations of the ground state of the linear Schrödinger equation. These solutions are off-set from the zero level. This is confirmed with extensive numerical simulations on the governing nonlinear equation. Likewise, nontrivial-phase solutions are stable if they are sufficiently off-set. Thus for condensates with repulsive interactions, a large number of condensed atoms is sufficient to form a stable, periodic condensate, as in the one-dimensional case [10]. Physically, this implies stability of states near the Thomas-Fermi limit.

For condensates with attractive atomic interactions, no stable solutions are found, in contrast to the one-dimensional case [8]. However, the time scale for the onset of instability for some of our solutions far exceeds the time scale of current physical experiments with attractive condensates. This occurs when the solutions are localized in the troughs of the potential and have nodes. These solutions may be observable in experiments, given the time scale over which the instability develops.

Many issues remain open. In particular, for the majority of our solutions, we have no analytical results concerning stability or instability. Nontrivial phase solutions especially have eluded analysis. A more detailed understanding of the collapse and blow up in periodic potentials is necessary. Of particular interest is the full class of initial conditions which lead to collapse and blow-up [31, 35]. Furthermore, the effect of a confining potential in addition to a periodic potential warrants investigation. These are only a sampling of the many mathematical and physical issues which arise in this interesting problem. 


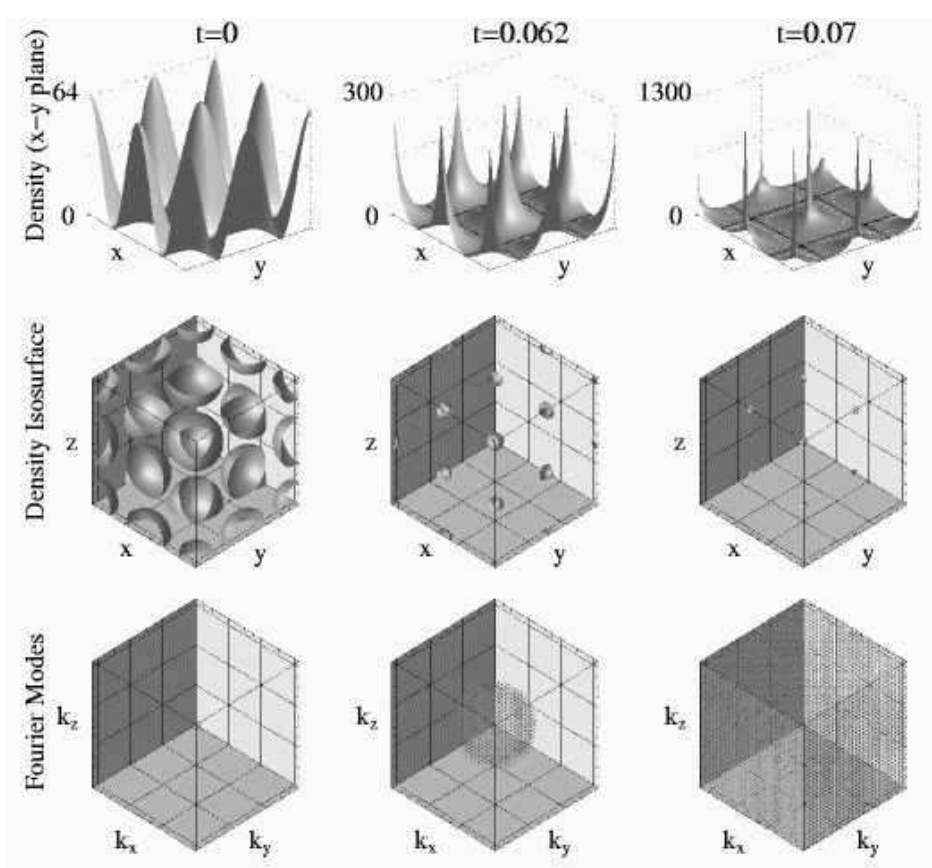

Figure 26: The three-dimensional unstable attractive evolution of a double-amplitude $\operatorname{cn}\left(m_{i} x_{i}, k_{i}\right)$ initial condition corresponding to (16c) with eight periods (two in each direction), with $k_{1}=k_{2}=$ $k_{3}=0.5, m_{1}=m_{2}=m_{3}=1$, and $A_{1}=A_{2}=A_{3}=-1$.
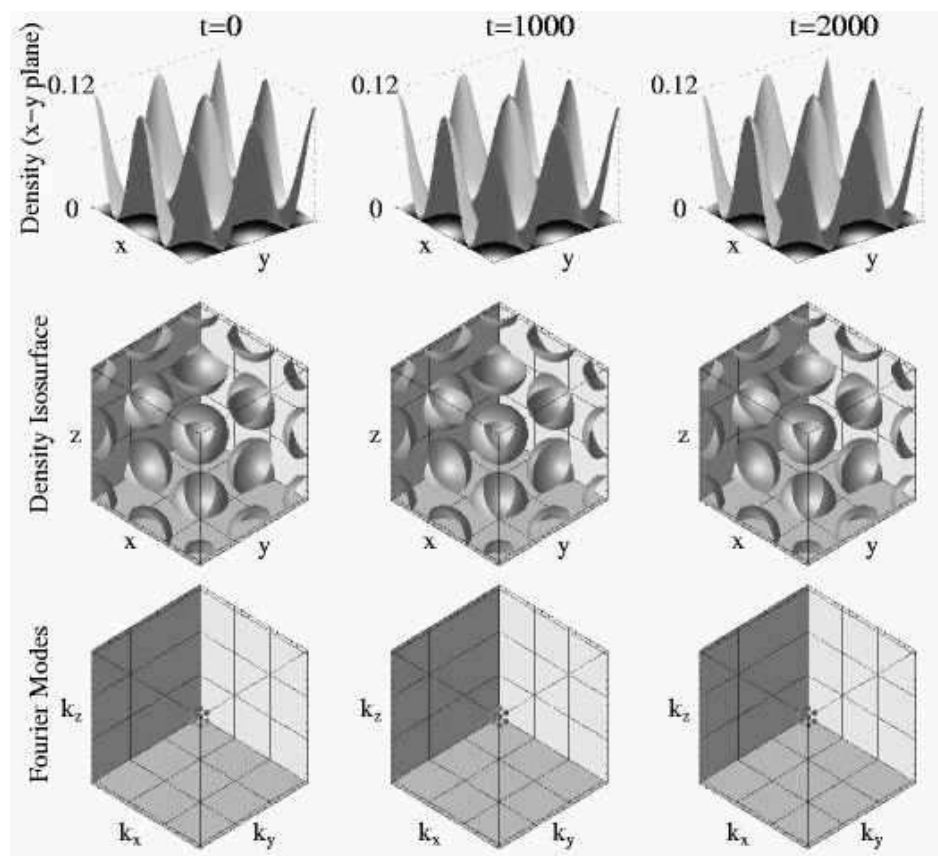

Figure 27: The three-dimensional stable attractive evolution of the exact $\operatorname{cn}\left(m_{i} x_{i}, k_{i}\right)$ solution corresponding to (16c) with eight periods (two in each direction), with $k_{1}=k_{2}=k_{3}=0.5$, $m_{1}=m_{2}=m_{3}=1$, and $A_{1}=A_{2}=A_{3}=-0.5$. 


\section{Acknowledgments}

The authors acknowledge useful conversations with J. C. Bronski and K. Promislow about the linear stability analysis and with W. P. Reinhardt concerning Table 1.

\section{Appendix A: The ground state of the Nonlinear Schrödinger equa- tion with repulsive atomic interaction}

This appendix is due to Jared C. Bronski.

Theorem 3 A stationary solution $\psi(\vec{x}, t)=r(\vec{x}) e^{i(\theta(\vec{x})-\omega t)}$ with $r(\vec{x})>0$ of (任), $\alpha=1$ is a global minimizer of the Hamiltonian (30) with the constraint that $\|\psi(x, t)\|^{2}=C$, for some constant $C$.

Proof Consider the reduced Hamiltonian

$$
\hat{\mathcal{H}}(\psi)=\mathcal{H}(\psi)-\lambda|| \psi \|^{2}=\int_{\Omega}\left(\frac{1}{2} \nabla \psi \cdot \nabla \psi^{*}+V(\vec{x})|\psi|^{2}+\frac{1}{2}|\psi|^{4}-\lambda|\psi|^{2}\right) d \vec{x},
$$

where $\lambda$ is a Lagrange multiplier. Its critical points are determined by

$$
\delta \hat{\mathcal{H}}(\psi)=0 \Rightarrow \lambda \psi=-\frac{1}{2} \Delta \psi+|\psi|^{2} \psi+V(\vec{x}) \psi .
$$

Thus the Lagrange multiplier $\lambda$, which enforces the constraint of fixed particle number, is interpreted physically as the chemical potential $\omega$. In what follows, the factor $e^{-i \omega t}$ in the solution is omitted. In general, an expression for $\lambda=\omega$ in terms of the critical point $\psi$ is obtained by multiplying this expression by $\psi^{*}$ and integrating over $\Omega$ :

$$
\omega=\frac{\int_{\Omega}\left(\frac{1}{2} \nabla \psi \cdot \nabla \psi^{*}+V(\vec{x})|\psi|^{2}+|\psi|^{4}\right) d \vec{x}}{\|\psi\|^{2}} .
$$

Global minimizers of $\mathcal{H}(\psi)$ subject to the constraint $\|\psi\|^{2}=C$ may be assumed to be real, since

$$
\begin{aligned}
\left\|r(\vec{x}) e^{i \theta(\vec{x})}\right\|^{2} & =\|r(\vec{x})\|^{2} \\
\mathcal{H}\left(r(\vec{x}) e^{i \theta(\vec{x})}\right) & =\mathcal{H}(r(\vec{x}))+\frac{1}{2} \int_{\Omega} r^{2}(\nabla \theta)^{2} d \vec{x} \geq \mathcal{H}(r(\vec{x})),
\end{aligned}
$$

with equality only valid for $\nabla \theta=0$. In this case, because of the phase invariance of (1), we can choose $\theta=0$. Clearly a global minimizer is only fixed up to multiplication by a constant phase factor.

The second variation of the reduced Hamiltonian is given by the quadratic form

$$
\begin{aligned}
\left\langle\phi\left|\delta^{2} \hat{\mathcal{H}}(\psi)\right| \phi\right\rangle & =\int_{\Omega}\left(\frac{1}{2} \nabla \phi \cdot \nabla \phi^{*}+V(\vec{x})|\phi|^{2}+\frac{1}{2} \psi^{2} \phi^{* 2}+\frac{1}{2} \psi^{* 2} \phi^{2}+2|\psi|^{2}|\phi|^{2}-\omega|\phi|^{2}\right) d \vec{x} \\
& =\left\langle u\left|L_{+}\right| u\right\rangle+\left\langle v\left|L_{-}\right| v\right\rangle,
\end{aligned}
$$

where $\psi$ is assumed to be real, $\phi=u+i v$, and $L_{+}$and $L_{-}$are defined by (21a-b) with $\theta=0$. From the proof of Theorem 1, it follows that for $\psi(\vec{x})=r(\vec{x})>0$, the operator $L_{+}$is positive definite, whereas $L_{-}$is positive semi-definite. Thus $\psi(\vec{x})=r(\vec{x})>0$ is a local minimizer of the Hamiltonian, subject to the constraint $\|\psi\|^{2}=C$. 
The proof that a positive solution is necessarily a global minimizer is by contradiction. Assume there exists a $\hat{\psi}$ such that $\|\hat{\psi}\|^{2}=C$ and $\mathcal{H}(\hat{\psi})<\mathcal{H}(\psi)$. By assumption, $\psi$ is real. This can also be assumed for $\hat{\psi}$ since this can only lower the energy. Now consider

$$
\Psi(\nu)=\cos (\nu) \psi+i \sin (\nu) \hat{\psi}
$$

Then $\Psi(\nu)$ satisfies the constraint $\|\Psi(\nu)\|^{2}=C$, since

$$
\|\Psi(\nu)\|^{2}=\cos ^{2}(\nu)\|\psi\|^{2}+\sin ^{2}(\nu)\|\hat{\psi}\|^{2}=C .
$$

Also,

$$
\begin{aligned}
\mathcal{H}(\Psi(\nu))= & \cos ^{2}(\nu) \int_{\Omega}\left(\frac{1}{2} \nabla \psi \cdot \nabla \psi^{*}+V(\vec{x})|\psi|^{2}+\frac{1}{2} \cos ^{2}(\nu)|\psi|^{4}\right) d \vec{x}+ \\
& \sin ^{2}(\nu) \int_{\Omega}\left(\frac{1}{2} \nabla \hat{\psi} \cdot \nabla \hat{\psi}^{*}+V(\vec{x})|\hat{\psi}|^{2}+\frac{1}{2} \sin ^{2}(\nu)|\hat{\psi}|^{4}\right) d \vec{x}+ \\
& \cos ^{2}(\nu) \sin ^{2}(\nu) \int_{\Omega}|\psi|^{2}|\hat{\psi}|^{2} d \vec{x} \\
\leq & \cos ^{2}(\nu) \mathcal{H}(\psi)+\sin ^{2}(\nu) \mathcal{H}(\hat{\psi})
\end{aligned}
$$

This last inequality follows from $\int_{\Omega}\left(\cos ^{4}(\nu)|\psi|^{4}+2 \cos ^{2}(\nu) \sin ^{2}(\nu)|\psi|^{2}|\hat{\psi}|^{2}+\sin ^{4}(\nu)|\hat{\psi}|^{4}\right) d \vec{x} \leq$ $\int_{\Omega}\left(\cos ^{2}(\nu)|\psi|^{4}+\sin ^{2}(\nu)|\hat{\psi}|^{4}\right) d \vec{x}$, which is easily verified. Equation (38) expresses the convexity of the Hamiltonian as a functional of $\psi$. Now, since $\mathcal{H}(\hat{\psi})<\mathcal{H}(\psi)$, near $\nu=0$

$$
\begin{aligned}
\mathcal{H}(\Psi(\nu)) & \leq\left(1-\frac{\nu^{2}}{2}+\mathcal{O}\left(\nu^{4}\right)\right)^{2} \mathcal{H}(\psi)+\left(\nu-\frac{\nu^{3}}{6}+\mathcal{O}\left(\nu^{5}\right)\right)^{2} \mathcal{H}(\hat{\psi}) \\
& \leq \mathcal{H}(\psi)-\nu^{2}(\mathcal{H}(\psi)-\mathcal{H}(\hat{\psi}))+\mathcal{O}\left(\nu^{4}\right),
\end{aligned}
$$

and thus $\mathcal{H}(\Psi(\nu))$ is quadratically decreasing near $\nu=0$. But,

$$
\begin{aligned}
\left.\frac{1}{2} \frac{d^{2}}{d \nu^{2}} \mathcal{H}(\Psi(\nu))\right|_{\nu=0}= & -\int_{\Omega}\left(\frac{1}{2} \nabla \psi \cdot \nabla \psi^{*}+V(\vec{x})|\psi|^{2}+|\psi|^{4}\right) d \vec{x}+ \\
& \int_{\Omega}\left(\frac{1}{2} \nabla \hat{\psi} \cdot \nabla \hat{\psi}^{*}+V(\vec{x})|\hat{\psi}|^{2}+|\psi|^{2}|\hat{\psi}|^{2}\right) d \vec{x} \\
= & \int_{\Omega}\left(\frac{1}{2} \nabla \hat{\psi} \cdot \nabla \hat{\psi}^{*}+V(\vec{x})|\hat{\psi}|^{2}+|\psi|^{2}|\hat{\psi}|^{2}\right) d \vec{x}-\omega \int_{\Omega}|\psi|^{2} d \vec{x} \\
= & \left\langle\hat{\psi}\left|L_{-}\right| \hat{\psi}\right\rangle \geq 0,
\end{aligned}
$$

where we have used (33). This contradicts the quadratic decrease of $\mathcal{H}(\Psi(\nu))$ near $\nu=0$. Thus, no $\hat{\psi}$ exists which satisfies $\|\hat{\psi}\|^{2}=C$ and which has a lower energy value than $\psi$. This proves the theorem.

\section{Appendix B: Collapse and blow-up}

A comprehensive overview of many aspects of collapse and blow-up for the attractive $(\alpha=-1)$ nonlinear Schrödinger equation (11) with $V(\vec{x})=0$ is given in [33. However, some modification 
of the theory is required for nonzero potential $V(\vec{x}) \neq 0$. In [29, 34 such modifications were considered for harmonic trap potentials. However, part of the analysis given in [34 applies to general potentials. This is the starting point of our considerations.

For the remainder of this appendix, we consider condensates with attractive atomic interactions for which $\alpha=-1$. Equation (1) has two conserved quantities, given by the $L^{2}$-norm of the solution (this has an interpretation as the number of particles per period $N$ ) and the Hamiltonian (30). In quantum mechanics, the nonlinear term in (1) is absent, and $\psi(\vec{x}, t)$ has the interpretation of a probability density function. This allows the definition of the expected value of a physical quantity. For instance,

$$
\vec{X}=<\vec{x}>=\frac{1}{N} \int_{\Omega} \vec{x}|\psi|^{2} d \vec{x}
$$

is the expected value of the position. Such quantities can still be constructed in the presence of the nonlinearity, although some care is required to interpret them. The above definition leads to

$$
\frac{d^{2} \vec{X}}{d t^{2}}=-\langle\nabla V(\vec{x})\rangle
$$

This equation is Newton's law. In quantum mechanics, the expected values of physical quantities satisfy classical equations of motion. That this equation persists in the presence of a nonlinear term is surprising.

To examine collapse, the quantity [33]

$$
W(t)=\int_{\Omega}\left(\sum_{i=1}^{d} x_{i}^{2}\right)|\psi|^{2} d \vec{x}
$$

is studied. Note that it is by definition a positive quantity. The solution of (11) is said to collapse if $W(t)$ becomes negative [33, 34]. Use of the conserved quantities and Newton's law (42) gives the variance identities

$$
\begin{aligned}
\frac{d^{2} W}{d t^{2}} & =2 \int_{\Omega}|\psi|^{2} d \vec{x}-d \int_{\Omega}|\psi|^{4} d \vec{x}-2 \int_{\Omega}|\psi|^{2}(\vec{x} \cdot \nabla V(\vec{x})) d \vec{x} \\
& =4 \mathcal{H}(\psi)-(d-2) \int_{\Omega}|\psi|^{4} d \vec{x}-2 \int_{\Omega}|\psi|^{2}(2 V(\vec{x})+\vec{x} \cdot \nabla V(\vec{x})) d \vec{x}
\end{aligned}
$$

These identities are generalizations of variance identities for the free nonlinear Schrödinger equation $(V(\vec{x})=0)$. With $d \geq 2$,

$$
\frac{d^{2} W}{d t^{2}} \leq 4 \mathcal{H}(\psi)-2 \int_{\Omega}|\psi|^{2} G(\vec{x}) d \vec{x},
$$

with $G(\vec{x})=2 V(\vec{x})+\vec{x} \cdot \nabla V(\vec{x})$. For specific choices of the potential (such as a harmonic trap, for which $\Omega=\mathbb{R}^{d}$ ) a more detailed analysis of the collapse is possible. However, for the potential (3), the analysis here is restricted to general statements.

If $G(\vec{x}) \geq 0$ then

$$
\begin{gathered}
\frac{d^{2} W}{d t^{2}} \leq 4 \mathcal{H}(\psi) \\
\Rightarrow W(t) \leq 2 \mathcal{H}(\psi) t^{2}+\beta t+\gamma
\end{gathered}
$$

If for the initial condition $\mathcal{H}(\psi) \leq 0$, then for all $t, \mathcal{H}(\psi) \leq 0$, since the Hamiltonian is conserved. Then $d^{2} W / d t^{2} \leq 0$, and for some time $t_{c}, W(t)<0$ if $t>t_{c}$. A crude overestimate of this time $t_{c}$ 
is found from (47). Presumably it is possible for solutions with positive energy to collapse as well, but this requires more subtle arguments [34, 35] than those used here.

Thus, for a given potential one checks whether $G(\vec{x}) \geq 0$. If so, then every initial condition of (1) with negative energy collapses in finite time. Note that it is always possible to ensure $G(\vec{x}) \geq 0$ on $\Omega$ : this can be achieved by adding a constant $V_{0}$ to the potential $V(\vec{x})$, since $\Omega$ is a bounded domain. However, this increases the energy of the solution by an amount $N V_{0}$. Note that the absolute level of the potential is physically irrelevant, which is clear from (44a), where only the gradient of the potential appears. In order to maximize the set of initial conditions for which collapse can be concluded, the constant $V_{0}$ is chosen such that $G(\vec{x}) \geq 0$, where equality is achieved in $\Omega$.

The exact solutions constructed in Section 2 are stationary, thus $W(t)$ is constant for these solutions and $d^{2} W / d t^{2}=0$. Thus, no conclusion for these solutions follows from our analysis. By increasing the amplitude of the initial conditions, we no longer find exact stationary solutions. However, it is possible to show that these initial conditions lead to finite-time collapse. This is the approach followed for many of the numerical runs in two and three dimensions.

Finally, following [34], it is easy to show that collapse of a solution implies blow-up of that solution, i.e., $\sup _{\Omega}|\psi| \rightarrow \infty$. The argument here is easier than the argument in [34] because $\Omega$ is bounded.

Theorem 4 For condensates with attractive atomic interactions $(\alpha=-1)$, collapse implies blowup.

Proof Consider

$$
\int_{\Omega}|\psi|^{4} d \vec{x} \leq\left(\sup _{\Omega}|\psi|\right)^{2} \int_{\Omega}|\psi|^{2} d \vec{x}=N\left(\sup _{\Omega}|\psi|\right)^{2} .
$$

The Heisenberg uncertainty principle states

$$
W(t) \int_{\Omega} \nabla \psi \cdot \nabla \psi^{*} d \vec{x} \geq\left(\frac{d N}{2}\right)^{2},
$$

thus

$$
\begin{aligned}
\left(\sup _{\Omega}|\psi|\right)^{2} & \geq \frac{1}{N} \int_{\Omega}|\psi|^{4} d \vec{x} \\
& =\frac{1}{N}\left(-2 \mathcal{H}(\psi)+\int_{\Omega} \nabla \psi \cdot \nabla \psi^{*} d \vec{x}+2 \int_{\Omega} V(\vec{x})|\psi|^{2} d \vec{x}\right) \\
& \geq \frac{1}{N}\left(-2 \mathcal{H}(\psi)+\left(\frac{d N}{2}\right)^{2} \frac{1}{W}+2 \int_{\Omega} V(\vec{x})|\psi|^{2} d \vec{x}\right)
\end{aligned}
$$

Since $\int_{\Omega} V(\vec{x})|\psi|^{2} d \vec{x} \in\left[-N \sup _{\Omega} V(\vec{x}), N \sup _{\Omega} V(\vec{x})\right]$, this implies that as $W(t) \rightarrow 0, \sup _{\Omega}|\psi| \rightarrow \infty$.

\section{References}

[1] B. P. Anderson and M. A. Kasevich. Macroscopic quantum inteference from atomic tunnel arrays. Science, 282:1686-1689, 1998. 
[2] B. P. Anderson and M. A. Kasevich. Macroscopic quantum interference from atomic tunnel arrays. Science, 282:1686-89, 1998.

[3] N. H. Anderson, J. R. Ensher, M. R. Matthews, C. E. Wieman, and E. A. Cornell. Observation of Bose-Einstein condensation in a dilute atomic vapor. Science, 269:198-201, 1995.

[4] N. W. Ashcroft and N. D. Mermin. Solid State Physics. Saunders College Publishing, Philadelphia, 1976.

[5] K. Berg-Sørensen and K. Mølmer. Bose-Einstein condensates in spatially periodic potentials. Phys. Rev. A, 58:1480-1484, 1998.

[6] K. Bongs, S. Berger, S. Dettmer, D. Hellweg, J. Arlt, W. Ertmer, and K. Sengstock. A waveguide for Bose-Einstein condensates. cond-mat/0007381, 2000.

[7] G. K. Brennen, C. M. Caves, P. S. Jessen, and I. H. Deutsch. Quantum logic gates in optical lattices. Phys. Rev. Letters, 82(5):1060-63, 1999.

[8] J. C. Bronski, L. D. Carr, R. Carretero-González, B. Deconinck, and J. N. Kutz. Stability of attractive Bose-Einstein condensates in a periodic potential. Submitted for publication; cond-mat/0012096.

[9] J. C. Bronski, L. D. Carr, B. Deconinck, and J. N. Kutz. Bose-Einstein condensates in standing waves: the cubic nonlinear Schrödinger equation with a periodic potential. Phys. Rev. Lett., 86(8):1402-1405, 2001.

[10] J. C. Bronski, L. D. Carr, B. Deconinck, J. N. Kutz, and K. Promislow. Stability of repulsive Bose-Einstein condensates in a periodic potential. Phys. Rev. E, 63(036612), 2001.

[11] M. L. Chiofalo and M. P. Tosi. Output from Bose condensates in tunnel arrays: the role of mean-field interactions and of transverse confinement. Phys. Lett. A, 268:406-12, 2000.

[12] D.-I. Choi and Q. Niu. Bose-Einstein condensates in an optical lattice. Phys. Rev. Letters, 82(10):2022-25, 1999.

[13] D. I. Choi and Q. Niu. Bose-Einstein condensates in an optical lattice. Phys. Rev. Lett., 82:2022-2025, 1999.

[14] R. Courant and D. Hilbert. Methods of mathematical physics. Vol. I. Interscience Publishers, Inc., New York, N.Y., 1953.

[15] K. B. Davis, M.-O. Mewes, M. R. Andrews, N. J. van Druten, D. S. Durfee, D. M. Kurn, and W. Ketterle. Bose-Einstein condensation in a gas of sodium atoms. Phys. Rev. Lett., 78:3969-3973, 1995.

[16] B. Deconinck, B. A. Frigyik, and J. N. Kutz. Stability of exact solutions of the defocusing nonlinear Schrödinger equation with periodic potential in two dimensions. Phys. Lett. A, 2001. Accepted for publication.

[17] D. G. Fried, T. C. Killian, L. Willmann, D. Landhuis, S. C. Moss, D. Kleppner, and T. J. Greytak. Bose-Einstein condensation of atomic hydrogen. Phys. Rev. Lett., 81:3811-3814, 1998. 
[18] S. Gasiorowicz. Quantum Physics. John Wiley \& Sons, New York, 1974.

[19] E. P. Gross. Structure of a quantized vortex in boson systems. Nuovo Cimento, 20:454-477, 1961.

[20] E. W. Hagley, L. Deng, M. Kozuma, J. Wen, K. Helmerson, S. L. Rolston, and W. D. Phillips. A well-collimated quasi-continuous atom laser. Science, 283:1706-09, 1999.

[21] D.-J. Han, R. H. Wynar, Ph. Courteille, and D. J. Heinzen. Bose-Einstein condensation of large numbers of atoms in a magnetic time-averaged orbiting potential trap. Phys. Rev. A, 57:R4114-R4117, 1998.

[22] T. Y. Hou, J. S. Lowengrub, and M. J. Shelley. Removing the stiffness from interfacial flows with surface tension. J. Comput. Phys., 114:312-338, 1994.

[23] D. Jaksch, C. Bruder, J. I. Cirac, C. W. Gardiner, and P. Zoller. Cold bosonic atoms in optical lattices. Phys. Rev. Letters, 81(15):3108-3111, 1998.

[24] M. Kozuma, Y. Suzuki, T. Torii, Y. Sugiura, T. Kuga, E. W. Hagley, and L. Deng. Phasecoherent amplification of matter waves. Science, 286:2309-12, 1999.

[25] Y. B. Ovchinnikov, J. H. Müller, M. R. Doery, E. J. D. Vredenbregt, K. Helmerson, S. L. Rolston, and W. D. Phillips. Diffraction of a released Bose-Einstein condensate by a pulsed standing light wave. Phys. Rev. Letters, 83(2):284-87, 1999.

[26] D. S. Petrov, M. Holzmann, and G. V. Shlyapnikov. Bose-Einstein condensation in quasi-2D trapped gases. Phy. Rev. Lett., 84:2551-2555, 2000.

[27] D. S. Petrov, G. V. Shlyapnikov, and J. T. M. Walraven. Regimes of quantum degeneracy in trapped 1D gases. Phy. Rev. Lett., 85:3745-3749, 2000.

[28] L. P. Pitaevskii. Vortex lines in an imperfect bose gas. Sov. Phys. JETP, 13:451-454, 1961.

[29] L.P. Pitaevskii. Dynamics of collapse of a confined Bose gas. Phys. Lett. A, 221:14-18, 1996.

[30] J. L. Roberts, N. R. Claussen, S. L. Cornish, E. A. Donley, E. A. Cornell, and C. E. Wieman. Observation of Bose-Einstein condensation in a dilute atomic vapor. Phys. Rev. Lett., 86:20012004, 2001.

[31] Harvey A. Rose and Michael I. Weinstein. On the bound states of the nonlinear Schrödinger equation with a linear potential. Phys. D, 30(1-2):207-218, 1988.

[32] C. A. Sackett, Stoof H. T. C., and Hulet R. G. Bose-Einstein condensation of atomic hydrogen. Phys. Rev. Lett., 80:2031-2034, 1998.

[33] C. Sulem and P.-L. Sulem. The nonlinear Schrödinger equation: self-focusing and wave collapse. Springer-Verlag, New York, 1999.

[34] T Tsurumi, H. Morise, and M. Wadati. Stability of Bose-Einstein condensates confined in traps. International Journal of Modern Physics B, 14:655-719, 2000.

[35] M. I. Weinstein. Nonlinear Schrödinger equations and sharp interpolation estimates. Comm. Math. Phys., 87(4):567-576, 1982/83. 
[36] E. T. Whittaker and G. N. Watson. A Course in Modern Analysis. Cambridge University Press, Cambridge, 1963. 